\title{
TOEPLITZ QUANTIZATION AND ASYMPTOTIC EXPANSIONS FOR REAL BOUNDED SYMMETRIC DOMAINS
}

\author{
MIROSLAV ENGLIŠ AND HARALD UPMEIER
}

\begin{abstract}
An analogue of the star product, familiar from deformation quantization, is studied in the setting of real bounded symmetric domains. The analogue turns out to be a certain invariant operator, which one might call star restriction, from functions on the complexification of the domain into functions on the domain itself. In particular, we establish the usual (i.e. semiclassical) asymptotic expansion of this star restriction, and further obtain a real-variable analogue of a theorem of Arazy and Ørsted concerning the analogous expansion for the Berezin transform.
\end{abstract}

\section{Covariant Calculi on Complex and Real Symmetric Domains}

Let $D=G / K$ be an irreducible bounded symmetric domain in $\mathbf{C}^{d}$ in the HarishChandra realization, with $G$ the identity connected component of the group of all biholomorphic self-maps of $D$ and $K$ the stabilizer of the origin; $K$ can also be realized as the automorphism group $\operatorname{Aut}(Z)$ of the Hermitian Jordan triple $Z \approx \mathbf{C}^{d}$ associated with $D$. For $\nu>p-1, p$ being the genus of $D$, let $H_{\nu}^{2}(D)$ denote the standard weighted Bergman space on $D$, i.e. the subspace of all holomorphic functions in $L^{2}\left(D, d \mu_{\nu}\right)$, with

$$
d \mu_{\nu}(z)=c_{\nu} K(z, z)^{1-\nu / p} d z
$$

where $d z$ stands for the Lebesgue measure, $K(z, w)$ is the ordinary (unweighted) Bergman kernel of $D$, and $c_{\nu}$ is a normalizing constant to make $d \mu_{\nu}$ a probability measure. The space $H_{\nu}^{2}(D)$ carries the unitary representation $U^{(\nu)}$ of $G$ given by

$$
U_{g}^{(\nu)} f(z)=f\left(g^{-1}(z)\right) \cdot J_{g^{-1}}(z)^{\nu / p}, \quad g \in G, f \in H_{\nu}^{2}(D),
$$

where $J_{g}$ denotes the complex Jacobian of the mapping $g$. (In general, if $\nu / p$ is not an integer, then $U^{(\nu)}$ is only a projective representation due to the ambiguity in the choice of the power $J_{g^{-1}}(z)^{\nu / p}$.) This situation will henceforth be called the complex bounded case.

In addition to bounded symmetric domains, we will also consider the complex flat case of a Hermitian vector space $D=Z \approx \mathbf{C}^{d}$, with $D=G / K$ for $G$ the group of all orientation-preserving rigid motions of $Z$, and $K=U(Z) \approx U_{d}(\mathbf{C})$ the stabilizer of the origin in $G$; the spaces $H_{\nu}^{2}(Z)$ will then be the Segal-Bargmann spaces of all

1991 Mathematics Subject Classification. Primary 32M15; Secondary 46E22, 47B35, 53D55.

Key words and phrases. bounded symmetric domain, Toeplitz operator, star product, covariant quantization.

Research supported by GA ČR grant no. 201/06/0128 and AV ČR institutional research plan AV0Z10190503.

Research supported by GIF grant no. 696-17.6/2001. 
entire functions which are square-integrable with respect to the Gaussian measure

$$
d \mu_{\nu}(z)=\left(\frac{\nu}{\pi}\right)^{d} e^{-\nu\|z\|^{2}} d z
$$

and $U^{(\nu)}$ will be the usual Schrödinger representation. When treated simultaneously, both settings will be called the complex case.

By a covariant operator calculus, or covariant quantization, on $D$ one understands a mapping $\mathcal{A}: f \mapsto \mathcal{A}_{f}$ from functions on $D$ into operators on $H_{\nu}^{2}(D)$ which is $G$-covariant in the sense that

$$
\mathcal{A}_{f \circ g}=U_{g}^{(\nu) *} \mathcal{A}_{f} U_{g}^{(\nu)}, \quad \forall g \in G .
$$

In most cases, such calculi can be built by the recipe

$$
\mathcal{A}_{f}=\int_{D} f(\zeta) \mathcal{A}_{\zeta} d \mu_{0}(\zeta)
$$

where $d \mu_{0}$ is a $G$-invariant measure on $D$, and $\mathcal{A}_{\zeta}$ is a family of operators in $H_{\nu}^{2}(D)$ labelled by $\zeta \in D$ such that

$$
\mathcal{A}_{g(\zeta)}=U_{g}^{(\nu)} \mathcal{A}_{\zeta} U_{g}^{(\nu) *}, \quad \forall g \in G
$$

(One calls such a family a covariant operator field on $D$. One also usually normalizes the measure $d \mu_{0}$ so that $\mathcal{A}_{1}$ is the identity operator.) Note that in view of the transitivity of the action of $G$ on $D$, any covariant operator field is uniquely determined by its value $\mathcal{A}_{0}$ at the origin $\zeta=0$.

The best known examples of such calculi are the Toeplitz calculus $\mathcal{T}$ and the Weyl calculus $\mathcal{W}$, corresponding to $\mathcal{T}_{0}=\langle\cdot, \mathbf{1}\rangle \mathbf{1}$ (the projection onto the constants) and $\mathcal{W}_{0} f(z)=f(-z)$ (the reflection operator), respectively [2]. For the complex flat case, $\mathcal{W}$ is just the well-known Weyl calculus from the theory of pseudodifferential operators, see e.g. [12].

Given a covariant operator calculus $\mathcal{A}$, the associated star product $*$ on functions on $D$ is defined by

$$
\mathcal{A}_{f * g}=\mathcal{A}_{f} \mathcal{A}_{g}
$$

While $f * g$ is a well-defined object for some calculi (e.g. for $\mathcal{A}=\mathcal{W}$, at least on $\mathbf{C}^{d}$ and rank one symmetric domains, see [4]), in most cases (e.g. for $\mathcal{A}=\mathcal{T}$, the Toeplitz calculus), it makes sense only for very special functions $f, g$ and (7) is then usually understood as an equality of asymptotic expansions as the Wallach parameter $\nu$ tends to infinity. For instance, for $\mathcal{A}=\mathcal{T}$, it was shown in [6] that for any $f, g \in C^{\infty}(D)$ with compact support,

$$
\left\|\mathcal{T}_{f} \mathcal{T}_{g}-\mathcal{T}_{\sum_{j=0}^{N} \nu^{-j} C_{j}(f, g)}\right\|=O\left(\nu^{-N-1}\right)
$$

as $\nu \rightarrow \infty$, for some bilinear differential operators $C_{j}$ (not depending on $f, g$ and $\nu$ ). (The assumption of compact support can be relaxed, cf. [9].) We can thus define $f * g$ as the formal power series

$$
f * g:=\sum_{j=0}^{\infty} \nu^{-j} C_{j}(f, g) .
$$

Interpreting $\nu$ as the reciprocal of the Planck constant, we recover the BerezinToeplitz star product, which is of central importance in quantization on Kähler manifolds [5]. 
In this paper we study covariant functional calculi and asymptotic expansions for the more general real bounded symmetric domains which yield, up to a few exceptions, all Riemannian symmetric spaces of non-compact type. In order to introduce real symmetric domains, suppose $Z^{\mathbf{C}}$ is an irreducible Hermitian Jordan triple endowed with a (conjugate-linear) involution

$$
z \mapsto z^{\#}
$$

which preserves the Jordan triple product and therefore the unit ball $D^{\mathbf{C}}$ of $Z^{\mathbf{C}}$, i.e. $\left(D^{\mathbf{C}}\right)^{\#}=D^{\mathbf{C}}$. Defining the real forms

$$
\begin{aligned}
& Z:=\left\{z \in Z^{\mathbf{C}}: z^{\#}=z\right\} \\
& D:=\left\{z \in D^{\mathbf{C}}: z^{\#}=z\right\}=Z \cap D^{\mathbf{C}},
\end{aligned}
$$

we obtain a commuting diagram

$$
\begin{array}{ccc}
Z & \subset & Z^{\mathbf{C}} \\
\cup & & \cup \\
D & \subset & D^{\mathbf{C}} .
\end{array}
$$

For the groups $G^{\mathbf{C}}:=\operatorname{Aut}\left(D^{\mathbf{C}}\right), K^{\mathbf{C}}:=\operatorname{Aut}\left(Z^{\mathbf{C}}\right)$ the given involution (10) induces conjugations

$$
\begin{aligned}
g^{\#}(z):=\left(g\left(z^{\#}\right)\right)^{\#} & \left(g \in G^{\mathbf{C}}, z \in D^{\mathbf{C}}\right) \\
k^{\#} z & :=\left(k z^{\#}\right)^{\#} \quad\left(k \in K^{\mathbf{C}}, z \in Z^{\mathbf{C}}\right)
\end{aligned}
$$

with fixed point groups

$$
\begin{aligned}
& G:=\left\{g \in G^{\mathbf{C}}: g\left(z^{\#}\right)=g(z)^{\#}\right\} \\
& K:=\left\{k \in K^{\mathbf{C}}: k z^{\#}=(k z)^{\#}\right\}=G \cap K^{\mathbf{C}}
\end{aligned}
$$

acting on $D$ and $Z$, respectively. Again, we obtain a commuting diagram

$$
\begin{array}{ccc}
G & \subset & G^{\mathbf{C}} \\
\cup & & \cup \\
K & \subset & K^{\mathbf{C}} .
\end{array}
$$

In this situation $Z$ is an irreducible real Jordan triple, $G$ is a reductive Lie group (it may have a nontrivial center), and

$$
D=G / K
$$

is an irreducible real bounded symmetric domain. This setting will be called the real bounded case.

In this situation a covariant quantization on $D$ is a map $f \mapsto \mathcal{A}_{f}$ from $C^{\infty}(D)$ into $H_{\nu}^{2}\left(D^{\mathbf{C}}\right)$ such that

$$
\mathcal{A}_{f \circ g}=U_{g}^{(\nu) *} \mathcal{A}_{f}
$$

for all $g \in G$. The counterpart of the star product, associated to a covariant quantization $\mathcal{A}$ on $D$ and a covariant quantization $\mathcal{A}^{\mathrm{C}}$ on $D^{\mathrm{C}}$, is the star restriction

defined by

$$
\#: C^{\infty}\left(D^{\mathbf{C}}\right) \rightarrow C^{\infty}(D)
$$

$$
\mathcal{A}_{\# F}=\mathcal{A}_{F}^{\mathbf{C}} I
$$

where

$$
I(z)=K^{(\nu)}\left(z, z^{\#}\right)^{1 / 2}
$$


is the unique $G$-invariant holomorphic function on $D^{\mathbf{C}}$ satisfying $I(0)=1$. In addition, we will again consider the above construction also in the case of the SegalBargmann spaces for an involutive Hermitian vector space $Z^{\mathbf{C}} \approx \mathbf{C}^{d}$, with the ordinary complex conjugation as the involution $z \mapsto z^{\#}$; thus $D=Z \approx \mathbf{R}^{d}$. This setting will be called the real flat case, and both situations together are referred to as the real case.

In most cases, calculi satisfying (15) can again be constructed by the recipe

$$
\mathcal{A}_{f}=\int_{D} f(\zeta) \mathcal{A}_{\zeta} d \mu_{0}(\zeta)
$$

where $d \mu_{0}$ is the $G$-invariant measure in $D$, and $\mathcal{A}_{\zeta}$ is a family of holomorphic functions (not necessarily belonging to $H_{\nu}^{2}\left(D^{\mathbf{C}}\right)$ ) labelled by $\zeta \in D$ which is covariant in the sense that

$$
\mathcal{A}_{g(\zeta)}=U_{g}^{(\nu)} \mathcal{A}_{\zeta}, \quad \forall g \in G, \zeta \in D .
$$

As before, one usually normalizes $d \mu_{0}$ so that $\mathcal{A}_{\mathbf{1}}=I$. The prime example is now the real Toeplitz calculus $\mathcal{A}=\mathcal{T}$ corresponding to $\mathcal{A}_{0}=\mathbf{1}$ (the function constant one) [7], [20]; there is also a notion of real Weyl calculus, but it is more complicated [3].

Our first main result in this paper is the existence of the star restriction i.e. an analogue of the asymptotic expansion (9) of the star product - for the real Toeplitz calculus.

Theorem. There exist G-invariant differential operators $L_{n}$ from $C^{\infty}\left(D^{\mathbf{C}}\right)$ into $C^{\infty}(D)$ such that, in an appropriate sense (cf. Definition 2 below),

$$
\# F=\sum_{n=0}^{\infty} \nu^{-n} L_{n} F,
$$

where \# is the star restriction operator associated to the real Toeplitz calculus $\mathcal{T}$ on $D$ and the usual Toeplitz calculus $\mathcal{T}^{\mathbf{C}}$ on $D^{\mathbf{C}}$.

We also show that the operators $L_{n}$ have a rather special form, and give a recipe for computing them via a kind of Harish-Chandra isomorphism; see Sections 3-4. Here a crucial role is played by the fact that \# enjoys the factorization property

$$
\#(\bar{h} F)=\bar{h}(\# F)
$$

for any holomorphic function $h$ on $D^{\mathbf{C}}$

Before describing our second main result, we show that the complex case of operator calculi on a complex bounded symmetric domain $D$ from the beginning of this section can be recovered within the more general real framework. Define the underlying "real" domain

$$
D_{\mathbf{R}}:=\{(z, \bar{z}): z \in D\} \subset Z_{\mathbf{R}}:=\{(z, \bar{z}): z \in Z\},
$$

where the bar indicates that we consider the "conjugate" complex structure for the second component. The complexifications

$$
\begin{aligned}
& D_{\mathbf{R}}^{\mathbf{C}}=\{(z, \bar{w}): z, w \in D\}=D \times \bar{D} \\
& Z_{\mathbf{R}}^{\mathbf{C}}=\{(z, \bar{w}): z, w \in Z\}=Z \times \bar{Z}
\end{aligned}
$$

are endowed with the flip involution

$$
(z, \bar{w})^{\#}:=(w, \bar{z})
$$


having fixed points $D_{\mathbf{R}}$ and $Z_{\mathbf{R}}$, respectively. This can be summarized in the commuting diagram

$$
\begin{aligned}
& Z \approx Z_{\mathbf{R}} \subset Z_{\mathbf{R}}^{\mathbf{C}}=Z \times \bar{Z} \\
& \cup \\
& \cup \\
& \cup D_{\mathbf{R}} \subset D_{\mathbf{R}}^{\mathbf{C}}=D \times \bar{D},
\end{aligned}
$$

where $\approx$ denotes the "diagonal embedding" $z \mapsto(z, \bar{z})$. Putting

$$
\begin{aligned}
\bar{g}(\bar{z}) & :=\overline{g(z)} & (g \in G, z \in D), \\
\bar{k} \bar{z} & :=\overline{k z} \quad & (k \in K, z \in Z)
\end{aligned}
$$

we obtain groups

$$
\begin{aligned}
G_{\mathbf{R}} & :=\{(g, \bar{g}): g \in G\}, \\
K_{\mathbf{R}} & :=\{(k, \bar{k}): k \in K\}
\end{aligned}
$$

acting "diagonally" on $D_{\mathbf{R}}$ and $Z_{\mathbf{R}}$, respectively, thus "forgetting" the complex structure. The flip involution on

$$
\begin{aligned}
& G_{\mathbf{R}}^{\mathbf{C}}:=\left\{\left(g_{1}, \bar{g}_{2}\right): g_{1}, g_{2} \in G\right\}=G \times \bar{G}, \\
& K_{\mathbf{R}}^{\mathbf{C}}:=\left\{\left(k_{1}, \bar{k}_{2}\right): k_{1}, k_{2} \in K\right\}=K \times \bar{K}
\end{aligned}
$$

has fixed points $G_{\mathbf{R}}$ and $K_{\mathbf{R}}$, respectively. This can be summarized in the commuting diagram

$$
\begin{aligned}
& G \approx G_{\mathbf{R}} \subset G_{\mathbf{R}}^{\mathbf{C}}=G \times \bar{G} \\
& \cup \\
& \cup
\end{aligned}
$$

where $\approx$ denotes the "diagonal" embedding on the group level.

Since $H_{\nu}^{2}(D)$ is a reproducing kernel space (with reproducing kernel $K^{(\nu)}(x, y)=$ $\left.\left[K(x, y) / c_{p}\right]^{\nu / p}\right)$, any bounded linear operator on $H_{\nu}^{2}(D)$ is automatically an integral operator: namely,

$$
T f(z)=\int_{D} f(w) \widetilde{T}(z, w) d \mu_{\nu}(w)
$$

with

$$
\widetilde{T}(z, w)=\overline{\left(T^{*} K^{(\nu)}(\cdot, z)\right)(w)}=\left\langle T K^{(\nu)}(\cdot, w), K^{(\nu)}(\cdot, z)\right\rangle .
$$

This follows from the identity $T f(z)=\left\langle T f, K^{(\nu)}(\cdot, z)\right\rangle=\left\langle f, T^{*} K^{(\nu)}(\cdot, z)\right\rangle$. In this way, we may identify operators on $H_{\nu}^{2}(D)$ with (some) functions on $D \times D$, holomorphic in the first and anti-holomorphic in the second variable; that is, with holomorphic functions on $D \times \bar{D}$. Upon this identification, the covariant quantization rule $f \mapsto \mathcal{A}_{f}$ becomes simply a (densely defined) operator $f \mapsto \widetilde{\mathcal{A}}_{f}$ from $C^{\infty}\left(D_{\mathbf{R}}\right)$ into the Hilbert space

$$
H_{\nu}^{2}\left(D_{\mathbf{R}}^{\mathbf{C}}\right) \approx H_{\nu}^{2}(D) \otimes \overline{H_{\nu}^{2}(D)}
$$

corresponding to the Hilbert-Schmidt operators, and the covariance condition (4) means that $\widetilde{\mathcal{A}}$ is equivariant under $G_{\mathbf{R}} \approx G$, i.e. intertwines the $G$-action on the former with the diagonal $G$-action on the latter:

$$
\widetilde{\mathcal{A}}_{f \circ g}=\left(U_{g}^{(\nu) *} \otimes \overline{U_{g}^{(\nu)}}\right) \widetilde{\mathcal{A}}_{f},
$$

in analogy to (15). Similarly, upon taking $\mathcal{A}^{\mathrm{C}}=\mathcal{A} \otimes \mathcal{A}$, and identifying pairs $f, g$ of functions on $D$ with the function $F(x, y)=f(x) \overline{g(y)}$ on $D \times \bar{D}$, (16) reduces 
just to (7). Note, however, that in the complex case the complexification (22) is not irreducible, but of "product type". When interpreted within the real setting, the complex case will often have a subscript $\mathbf{R}$ in the notation (cf. (21)).

It turns out that the asymptotic expansion (20) is closely related to another important concept, namely the Berezin transform $B_{\nu}$ (see Section 5 below for the various definitions and notations). In the complex case, Arazy and Ørsted [1] showed that there is an asymptotic expansion

$$
B_{\mathbf{R}, \nu}=\sum_{\mathbf{m}} \frac{K^{\mathbf{m}}(\partial, \partial)}{(\nu)_{\mathbf{m}}},
$$

where $K^{\mathbf{m}}$ are certain $K$-invariant polynomials, and $(\nu)_{\mathbf{m}}$ are the multi-variable Pochhammer symbols of $\nu$, labelled by the signatures $\mathbf{m}$ from the Peter-Weyl decomposition. Our second main result in this paper is an analogue of this formula for the Berezin transform $B_{\nu}$ on a general irreducible real bounded symmetric domain $D$.

Theorem. The real Berezin transform has an asymptotic expansion, as $\nu \rightarrow+\infty$,

$$
B_{\nu}=\sum_{\mathbf{m}} \frac{\Phi_{\mathbf{C}}^{\mathbf{m}}}{\{\nu\}_{\mathbf{m}}}
$$

where $\Phi_{\mathbf{C}}^{\mathbf{m}}$ are certain $G$-invariant linear differential operators on $D$ and $\{\nu\}_{\mathbf{m}}$ are certain "Pochhammer" symbols, with $\mathbf{m}$ running over all partitions of length $r$, given by a completely explicit formula (with the exception of domains of type (A); cf. Theorem 14).

The proof of (20) is given in Section 3, after reviewing the necessary background material in Section 2. The proof of (31) appears in Section 5, after establishing some facts about $G$-invariant operators from $C^{\infty}\left(D^{\mathbf{C}}\right)$ into $C^{\infty}(D)$ in Section 4 . The last Section 6 contains some concluding remarks and open problems.

ACKNOWLEDGEMEnT. Much of this work was done while the authors were visiting the Erwin Schrödinger Institute for Mathematical Physics in Vienna; the support of ESI is gratefully acknowledged.

\section{Bounded Symmetric DOMAINS OF COMPLEX AND REAL TYPE}

In order to describe the fine structure of real bounded symmetric domains, we start with an irreducible Euclidean Jordan algebra $X$ [11] which is uniquely determined (up to isomorphism) by two numerical invariants: the rank $r$ and the characteristic multiplicity $a$, such that

$$
d_{X}:=\operatorname{dim}_{\mathbf{R}} X=r+\frac{r(r-1)}{2} a .
$$

The associated Gindikin-Koecher gamma-function is given by

$$
\Gamma(\lambda)=(2 \pi)^{r(r-1) a / 4} \prod_{j=1}^{r} \Gamma\left(\lambda_{j}-\frac{j-1}{2} a\right) .
$$

Consider first the real case. Here $Z$ is a real form of an irreducible involutive Hermitian Jordan triple $Z^{\mathbf{C}}$. Let $e=e^{\#} \in Z$ be a maximal tripotent and consider the Peirce decomposition [11]

$$
Z=U \oplus V, \quad U=Z_{1}(e), \quad V=Z_{1 / 2}(e) .
$$


Then $U$ is a semi-simple, not necessarily Euclidean, real Jordan algebra with unit element $e$, which can be further decomposed into the \pm -eigenspaces

$$
X=\left\{x \in U: x^{*}=x\right\}, \quad Y=\left\{y \in U: y^{*}=-y\right\}
$$

under the Jordan involution $z^{*}=\{e z e\}$ on $Z^{\mathrm{C}}$. It follows that

$$
Z=X \oplus Y \oplus V \text {. }
$$

One can show that $X$ is an irreducible Euclidean Jordan algebra with unit element $e$. Considered as a (maximal) tripotent in $Z^{\mathrm{C}}, e$ induces also a "complex" Peirce decomposition

$$
Z^{\mathbf{C}}=U^{\mathbf{C}} \oplus V^{\mathbf{C}}
$$

and we obtain another irreducible Euclidean Jordan algebra

$$
X_{\mathbf{C}}:=\left\{x \in U^{\mathbf{C}}: x^{*}=x\right\}
$$

which contains $X$ as a unital Jordan subalgebra and has the same complexification $U^{\mathbf{C}}$ as $U=X \oplus Y$. This can be summarized in the commuting diagram

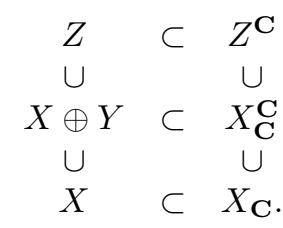

For the classification, one can distinguish three types, which reflect the different root systems for $D=G / K$. The domains of type (A) are realized as the unit ball $D$ of an irreducible Euclidean Jordan algebra $X$. In this case we have $Z=X$ and $Y=V=\{0\}$. Since $X=X_{\mathbf{C}}$, it follows that $r=r_{\mathbf{C}}, a=a_{\mathbf{C}}$. The following cases arise

$$
\begin{aligned}
a=1,2,4: & G L_{r}(\mathbf{K}) / U_{r}(\mathbf{K}) \subset \mathbf{K}_{\text {Herm }}^{r \times r} \\
& \text { for } \mathbf{K}=\mathbf{R}, \mathbf{C}, \mathbf{H} \text { (this is really the unbounded model) }, \\
a=8: & E_{6}^{(-26)} \times U_{1,1}(\mathbf{R}) / F_{4} \subset \mathbf{O}_{\mathrm{Herm}}^{3 \times 3}, \quad r=3 \\
a=d-2: & U_{1, d-1}(\mathbf{R}) / U_{d-1}(\mathbf{R}) \subset \mathbf{R}^{d}, r=2 \\
& \text { (the "algebra" real form of the complex spin factor). }
\end{aligned}
$$

For the remaining two types we have $Z \neq X \neq X_{\mathbf{C}}$. The type $(\mathbf{D} / \mathbf{B})$ is characterized by the conditions $r_{\mathbf{C}}=r, a_{\mathbf{C}}=2 a$. Up to one exception (root type $\left(D_{2}\right)$, which will not be considered here) it comprises the following cases

$$
\begin{array}{ll}
a=1: & U_{r, r+b}(\mathbf{R}) / U_{r}(\mathbf{R}) \times U_{r+b}(\mathbf{R}) \subset \mathbf{R}^{r \times(r+b)} \\
a=2: & O_{2 r+\varepsilon}(\mathbf{C}) / U_{2 r+\varepsilon}(\mathbf{R}) \subset \mathbf{R}_{\mathrm{asym}}^{(2 r+\varepsilon) \times(2 r+\varepsilon)}, \quad \varepsilon=0,1 \\
a=3: & U_{2,2}(\mathbf{H}) / U_{2}(\mathbf{H}) \times U_{2}(\mathbf{H}) \subset(\mathbf{H} \oplus \mathbf{H})^{1 \times 2}, \quad r=2 \\
a=4: & G L_{4}(\mathbf{H}) / U_{4}(\mathbf{H}) \subset(\mathbf{H} \oplus \mathbf{H})_{\text {Herm }}^{3 \times 3}, \quad r=3 .
\end{array}
$$

Here $\mathbf{H} \oplus \mathbf{H}$ denotes the split Cayley algebra.

Finally, the type $(\mathbf{C} / \mathbf{B C})$ is characterized by the conditions $r_{\mathbf{C}}=2 r, a_{\mathbf{C}}=a / 2$. (The latter equality makes sense only if $r>1$; for $r=1$ we actually take it as the definition of $a$.) It comprises the following cases:

$$
a=2: \quad S p_{2 r}(\mathbf{C}) / U_{r}(\mathbf{H}) \subset \mathbf{H}_{\mathrm{aHerm}}^{r \times r}
$$




$$
\begin{aligned}
a=4: & U_{r, r+b}(\mathbf{H}) / U_{r}(\mathbf{H}) \times U_{r+b}(\mathbf{H}) \subset \mathbf{H}^{r \times(r+b)} \\
a=12: & F_{4}^{(-20)} / S O(9) \subset \mathbf{O}^{1 \times 2}, \quad r=1 \\
a=2 d-4: & U_{1, d}(\mathbf{R}) / U_{d}(\mathbf{R}) \subset \mathbf{R}^{d}, \quad r=1
\end{aligned}
$$

(the "triple" real form of the complex spin factor).

Continuing with the real case let $G=N A K$ be the Iwasawa decomposition; for $g \in G$ we denote by $A(g) \in \mathfrak{a}$ the (unique) element of the Lie algebra $\mathfrak{a}$ of $A$ for which $g \in N \exp A(g) K$. Let further $M$ stand for the centralizer of $A$ in $K$. The conical functions $e^{\lambda, b}$ on $D$, where $\lambda \in \mathfrak{a}^{*} \otimes \mathbf{C}$ (the complexification of the dual $\mathfrak{a}^{*}$ of $\left.\mathfrak{a}\right)$ and $b \in K / M$, are defined by

$$
e^{\lambda, b}(g(0))=e^{\left\langle\lambda+\rho, A\left(b^{-1} g\right)\right\rangle}
$$

where $\rho$ denotes the half-sum of positive roots for $D \approx G / K$, explicitly given by

$$
\rho_{j}=\frac{a}{4}(2 j-r-1)+\frac{d_{Y}+d_{V} / 2}{2 r} \quad(1 \leq j \leq r) .
$$

(Here $d_{Y}$ and $d_{V}$ are the real dimensions of $Y$ and $V$, respectively.) The conical functions are joint eigenfunctions of all invariant differential operators. Namely, if $L$ is a linear differential operator on $D$ invariant under $G$ in the sense that

$$
L(f \circ g)=(L f) \circ g \quad \forall g \in G,
$$

then

$$
L e^{\lambda, b}=\widetilde{L}(\lambda) e^{\lambda, b} \quad \forall \lambda, b,
$$

for some $\widetilde{L}(\lambda) \in \mathbf{C}$ (independent of $b$ ). The mapping $L \mapsto \widetilde{L}$ is an isomorphism of the algebra of all invariant differential operators on $D$ onto the algebra of all polynomials on $\mathfrak{a}$ invariant under the action of the Weyl group (the Harish-Chandra isomorphism). This assertion remains in force also for more general operators than the differential ones; in particular, if $L$ is any $G$-invariant operator on some function space containing all the $e^{\lambda, b}$, then (43) still holds.

Important examples of such operators are the link transforms associated to covariant quantizations on $D$. For any covariant quantization $\mathcal{A}$ on $D$, its adjoint $\mathcal{A}^{*}$ acting from $H_{\nu}^{2}\left(D^{\mathbf{C}}\right)$ into functions on $D$ is given by

$$
\mathcal{A}^{*} f(x)=\left\langle f, \mathcal{A}_{x}\right\rangle_{\nu}
$$

and the composition $\mathcal{A}^{*} \mathcal{A}$ is a $G$-invariant operator on $D$. For the real Toeplitz calculus $\mathcal{A}=\mathcal{T}$, this link transform is known as the (real) Berezin transform $B_{\nu}$, and its eigenvalues $\widetilde{\mathcal{T}^{*} \mathcal{T}}(\lambda)=: b^{\nu}(\lambda)$ have been computed in [7], [20], [3], [26]:

$$
b^{\nu}(\lambda)=\frac{\Gamma\left(\nu_{\mathbf{R}}+\lambda+\rho-\frac{d_{Y}+d_{V} / 2}{r}\right) \Gamma\left(\nu_{\mathbf{R}}-\lambda+\rho-\frac{d_{Y}+d_{V} / 2}{r}\right)}{\Gamma\left(\nu_{\mathbf{R}}\right) \Gamma\left(\nu_{\mathbf{R}}-\frac{d_{Y}+d_{V} / 2}{r}\right)} .
$$

where

$$
\nu_{\mathbf{R}}:=\frac{\nu r_{\mathbf{C}}}{2 r} .
$$

In the flat case, the role of the conical functions is played by the ordinary exponentials

$$
e^{a}(x):=e^{\langle a, x\rangle}, \quad x \in Z=\mathbf{R}^{d}, \quad a \in Z^{\mathbf{C}}=\mathbf{C}^{d} ;
$$


invariant differential operators are just polynomials in the Laplacian, and

$$
\Delta e^{a}=\langle a, \bar{a}\rangle e^{a} .
$$

Finally, the Berezin transforms turn out to be just the familiar heat operators

$$
B_{\nu}=e^{\Delta / 2 \nu}
$$

so that

$$
b^{\nu}(a)=e^{\langle a, \bar{a}\rangle / 2 \nu} .
$$

This concludes our description of the real case. For the complex case, the situation is similar. We start with the (complex) Peirce decomposition

$$
Z=U \oplus V
$$

of an irreducible Hermitian Jordan triple $Z$ under a maximal tripotent $e \in Z$. Then

$$
X:=\left\{x \in U: x^{*}=x\right\}
$$

is an irreducible Euclidean Jordan algebra with unit element $e$ and complexification $X^{\mathbf{C}}=U$. Putting $Y:=i X$, we have

$$
Z=X^{\mathbf{C}} \oplus V=X \oplus Y \oplus V .
$$

The doubling process yields a commuting diagram

$$
\begin{aligned}
& Z \approx Z_{\mathbf{R}} \subset Z_{\mathbf{R}}^{\mathbf{C}}=Z \times \bar{Z} \\
& X^{\mathbf{C}} \approx X_{\mathbf{R}}^{\mathbf{C}} \subset X_{\mathbf{C}}^{\mathbf{C}}=X^{\mathbf{C}} \times \bar{X}^{\mathbf{C}} \text {, } \\
& \cup \stackrel{\cup}{\cup} \underset{X_{\mathbf{R}}}{U} \stackrel{\cup}{X_{\mathbf{C}}}:=X \times \bar{X}
\end{aligned}
$$

where $\approx$ denotes diagonal embedding and

$$
X_{\mathbf{C}}:=\{(x, \bar{y}): x, y \in X\}=X \times \bar{X}
$$

is a Euclidean Jordan algebra of product type, with unit element $(e, \bar{e})$, which has the same complexification as $X_{\mathbf{R}}^{\mathbf{C}}:=\{(x+i y, \overline{x+i y}): x, y \in X\}$. It follows that $r_{\mathbf{C}}=2 r, a_{\mathbf{C}}=a$. For the classification the following cases arise:

$$
\begin{array}{rll}
a=1: & S p_{2 r}(\mathbf{R}) / U_{r}(\mathbf{C}) \subset \mathbf{C}_{\mathrm{sym}}^{r \times r} \\
a=2: & U_{r, r+b}(\mathbf{C}) / U_{r}(\mathbf{C}) \times U_{r+b}(\mathbf{C}) \subset \mathbf{C}^{r \times(r+b)} \\
a=4: & O_{2 r+\varepsilon}(\mathbf{H}) / U_{2 r+\varepsilon}(\mathbf{C}) \subset \mathbf{C}_{\mathrm{asym}}^{(2 r+\varepsilon) \times(2 r+\varepsilon)}, \quad \varepsilon=0,1 \\
a=6: & E_{6}^{(-14)} / \mathbf{T} \times U_{10}(\mathbf{R}) \subset\left(\mathbf{O}^{\mathbf{C}}\right)^{1 \times 2}, \quad r=2 \\
a=8: & E_{7}^{(-25)} / \mathbf{T} \times E_{6} \subset\left(\mathbf{O}_{\mathrm{Herm}}^{3 \times 3}\right)^{\mathbf{C}}, \quad r=3 \\
a=d-2: & U_{2, d}(\mathbf{R}) / \mathbf{T} \times U_{d}(\mathbf{R}) \subset \mathbf{C}^{d}, r=2 \\
& (\text { complex spin factor }) .
\end{array}
$$

The group $O_{2 r+\varepsilon}(\mathbf{H})$ is usually denoted by $S O^{*}(4 r+2 \varepsilon)$.

Continuing with the complex case, let $G_{\mathbf{R}}=N_{\mathbf{R}} A_{\mathbf{R}} K_{\mathbf{R}}$ be the Iwasawa decomposition of $G_{\mathbf{R}} \approx G$. For $g \in G_{\mathbf{R}}$ we denote by $A(g) \in \mathfrak{a}_{\mathbf{R}}$ the (unique) element of the Lie algebra $\mathfrak{a}_{\mathbf{R}}$ of $A_{\mathbf{R}}$ for which $g \in N_{\mathbf{R}} \exp A(g) K_{\mathbf{R}}$. Let further $M_{\mathbf{R}}$ stand for 
the centralizer of $A_{\mathbf{R}}$ in $K_{\mathbf{R}}$. The conical functions $e_{\mathbf{R}}^{\lambda, b}$ on $D_{\mathbf{R}}$, where $\lambda \in \mathfrak{a}_{\mathbf{R}}{ }^{*} \otimes \mathbf{C}$ (the complexification of the dual of $\mathfrak{a}_{\mathbf{R}}$ ) and $b \in K_{\mathbf{R}} / M_{\mathbf{R}}$, are defined by

$$
e_{\mathbf{R}}^{\lambda, b}(g(0))=e^{\left\langle\lambda+\rho_{\mathbf{R}}, A\left(b^{-1} g\right)\right\rangle}
$$

where $\rho_{\mathbf{R}}$ is the half-sum of positive roots for $D_{\mathbf{R}} \approx G_{\mathbf{R}} / K_{\mathbf{R}}$, explicitly given by

$$
\left(\rho_{\mathbf{R}}\right)_{j}=\frac{a}{4}(2 j-r-1)+\frac{d}{4 r} \quad(1 \leq j \leq r) .
$$

The conical functions are joint eigenfunctions of all invariant differential operators. Namely, if $L$ is a linear differential operator on $D_{\mathbf{R}}$ invariant under $G_{\mathbf{R}}$ in the sense that

$$
L(f \circ g)=(L f) \circ g \quad \forall g \in G,
$$

then

$$
L e_{\mathbf{R}}^{\lambda, b}=\widetilde{L}(\lambda) e_{\mathbf{R}}^{\lambda, b} \quad \forall \lambda, b,
$$

for some $\widetilde{L}(\lambda) \in \mathbf{C}$ (independent of $b$ ). The mapping $L \mapsto \widetilde{L}$ is an isomorphism of the algebra of all invariant differential operators on $D_{\mathbf{R}}$ onto the algebra of all polynomials on $\mathfrak{a}_{\mathbf{R}}$ invariant under the action of the Weyl group (the Harish-Chandra isomorphism). This assertion remains in force also for more general operators than the differential ones; in particular, if $L$ is any $G_{\mathbf{R}}$-invariant operator on some function space containing all the $e_{\mathbf{R}}^{\lambda, b}$, then (58) still holds.

Important examples of such operators are the link transforms associated to covariant quantizations on $D$. Namely, for any covariant quantization $\mathcal{A}: f \mapsto \mathcal{A}_{f}$ from functions on $D$ into operators on $H_{\nu}^{2}(D)$, we can consider its formal adjoint $\mathcal{A}^{*}$ with respect to the $L^{2}\left(D_{\mathbf{R}}, d \mu_{0}\right)$ product of functions and the Hilbert-Schmidt inner product of operators. (See [10] for the discussion of the continuity etc. of $\mathcal{A}$ and $\mathcal{A}^{*}$.) A short computation [2] reveals that $\mathcal{A}^{*}$ is given by

$$
\mathcal{A}^{*} T(z)=\operatorname{tr}\left(T \mathcal{A}_{z}^{*}\right),
$$

and the $G_{\mathbf{R}}$-invariance of $\mathcal{A}$ translates into the property

$$
\mathcal{A}^{*}\left(U_{g}^{(\nu) *} T U_{g}^{(\nu)}\right)=\left(\mathcal{A}^{*} T\right) \circ g .
$$

(Here the $\mathcal{A}_{z}^{*}$ in (59) denotes the ordinary Hilbert space adjoint of the operator $\mathcal{A}_{z}$ on $H_{\nu}^{2}(D)$.) The composition $\mathcal{A}^{*} \mathcal{A}$ is therefore a $G_{\mathbf{R}}$-invariant operator on functions on $D_{\mathbf{R}}$, called the link transform corresponding to $\mathcal{A}$ :

$$
\mathcal{A}^{*} \mathcal{A}_{f \circ g}=\left(\mathcal{A}^{*} \mathcal{A}_{f}\right) \circ g, \quad \forall g \in G_{\mathbf{R}} .
$$

By (58), we thus have

$$
\mathcal{A}^{*} \mathcal{A}_{e_{\mathbf{R}}^{\lambda, b}}=\widetilde{\mathcal{A}^{*} \mathcal{A}}(\lambda) e_{\mathbf{R}}^{\lambda, b},
$$

for some Weyl-group-invariant function $\widetilde{\mathcal{A}^{*} \mathcal{A}}$ on $\mathfrak{a}_{\mathbf{R}}^{\mathbf{C}}$.

For the particular case of $\mathcal{A}=\mathcal{T}$, the Toeplitz calculus, the link transform $\mathcal{T}^{*} \mathcal{T}$ is known as the Berezin transform $B_{\mathbf{R}, \nu}$, and the function $\widetilde{\mathcal{T} * \mathcal{T}}=: b_{\mathbf{R}}^{\nu}$ has been computed in [24]:

$$
b_{\mathbf{R}}^{\nu}(\lambda)=\frac{\Gamma\left(\nu+\lambda+\rho_{\mathbf{R}}-d / r\right) \Gamma\left(\nu-\lambda+\rho_{\mathbf{R}}-d / r\right)}{\Gamma(\nu) \Gamma(\nu-d / r)} .
$$

This is analogous to (44), since $\nu_{\mathbf{R}}=\nu$ and $d_{V}=\frac{1}{2} d_{V_{\mathbf{R}}}$. 
In the complex flat case, the role of the conical functions is played by the ordinary exponentials

$$
e_{\mathbf{R}}^{a, b}(z):=e^{\langle a, z\rangle+\langle z, b\rangle}, \quad z, a, b \in Z=\mathbf{C}^{d},
$$

invariant differential operators are just polynomials in the Laplacian, and

$$
\Delta e_{\mathbf{R}}^{a, b}=4\langle a, b\rangle e_{\mathbf{R}}^{a, b},
$$

Finally, the Berezin transforms turn out to be just the familiar heat operators

$$
B_{\mathbf{R}, \nu}=e^{\Delta / 4 \nu},
$$

so that

$$
b_{\mathbf{R}}^{\nu}(a, b)=e^{\langle a, b\rangle / \nu} .
$$

We conclude this section by reviewing some facts about Bergman kernels on the complex domains. Let $D=G / K$ be an irreducible complex bounded symmetric domain in $Z \cong \mathbf{C}^{d}$, with rank $r$ and genus $p$. Fixing a Jordan frame $e_{1}, \ldots, e_{r}$, any element $z \in Z$ has a polar decomposition

$$
z=k \sum_{j} t_{j} e_{j}, \quad k \in K, t_{1} \geq t_{2} \geq \cdots \geq t_{r} \geq 0 .
$$

The numbers $t_{j}$ are uniquely determined by $z$, and $z \in D$ if and only if $t_{1}<1$.

The ordinary (unweighted) Bergman kernel $K(x, y)$ of $D$ has the form

$$
K(x, y)=c_{p} h(x, y)^{-p}, \quad x, y \in D,
$$

where $h(x, y)$, the Jordan determinant, is an irreducible polynomial, holomorphic in $x$ and $\bar{y}$, which is $K$-invariant in the sense that $h(k(x), k(y))=h(x, y) \forall k \in K$, and is uniquely determined by the property

$$
h(z, z)=\prod_{j}\left(1-t_{j}^{2}\right)
$$

for $z$ of the form (68). The weighted Bergman kernels with respect to the measures (1) are given by

$$
K^{(\nu)}(x, y)=h(x, y)^{-\nu} .
$$

For a Hermitian vector space $Z \approx \mathbf{C}^{d}$ the reproducing kernels of the spaces $H_{\nu}^{2}(Z)$ for the Gaussians (3) are

$$
K^{(\nu)}(x, y)=e^{\nu\langle x, y\rangle}, \quad x, y \in \mathbf{C}^{d} .
$$

The Fock inner product on the space $\mathcal{P}(Z)$ of all holomorphic polynomials on a Hermitian vector space $Z$ is defined as

$$
\langle p, q\rangle_{F}=\pi^{-d} \int_{Z} p(z) \overline{q(z)} e^{-\|z\|^{2}} d z=\left(\partial_{q} p\right)(0) .
$$

Here $\partial_{q}:=q^{*}(\partial), q^{*}(z):=\overline{q\left(z^{\#}\right)}$, is the constant coefficient (holomorphic) differential operator on $Z$ induced by $q \in \mathcal{P}(Z)$ via the scalar product (this depends conjugate-linearly on $q$ ). As a special case let $Z$ be an irreducible Hermitian Jordan triple of rank $r$, endowed with a normalized inner product which is invariant under the Jordan triple automorphism group $K=\operatorname{Aut}(Z)$. Under the action $p \mapsto p \circ k$, 
$k \in K$, of the compact group $K$ the space $\mathcal{P}(Z)$ has a Peter-Weyl decomposition into irreducible orthogonal components

$$
\mathcal{P}(Z)=\bigoplus_{\mathbf{m}} \mathcal{P}^{\mathbf{m}}(Z)
$$

where the summation extends over all signatures or partitions, i.e. $r$-tuples $\mathbf{m}=$ $\left(m_{1}, \ldots, m_{r}\right)$ of integers satisfying $m_{1} \geq m_{2} \geq \cdots \geq m_{r} \geq 0$. The polynomials in $\mathcal{P}^{\mathbf{m}}(Z)$ are homogeneous of degree $|\mathbf{m}|:=m_{1}+\cdots+m_{r}$. Each $\mathcal{P}^{\mathbf{m}}(Z)$, being a finite-dimensional space, has automatically bounded point evaluations at all points $z \in Z$, and thus $\mathcal{P}^{\mathbf{m}}(Z)$ has a reproducing kernel $K^{\mathbf{m}}(z, w), z, w \in Z$. The FarautKorányi formula asserts that the reproducing kernels $K^{(\nu)}(z, w)$ of $H_{\nu}^{2}(D)$ can be expressed in terms of these $K^{\mathbf{m}}$ by

$$
h(z, w)^{-\nu}=\sum_{\mathbf{m}}(\nu)_{\mathbf{m}} K^{\mathbf{m}}(z, w) .
$$

Here $(\nu)_{\mathbf{m}}$ are the generalized Pochhammer symbols

$$
(\nu)_{\mathbf{m}}:=\Gamma(\nu+\mathbf{m}) / \Gamma(\nu)=\prod_{j=1}^{r}\left(\nu-\frac{j-1}{2} a\right)_{m_{j}},
$$

where $(\nu)_{k}=\nu(\nu+1) \ldots(\nu+k-1)$ stands for the ordinary Pochhammer symbol.

For the complex flat case $Z \approx \mathbf{C}^{d}$, the Peter-Weyl decomposition under the unitary group $K=U(Z) \approx U_{d}(\mathbf{C})$ reduces to the homogeneous decomposition

$\mathcal{P}_{m}(Z)=\{$ homogeneous polynomials of degree $m\}$,

the reproducing kernels are given by $K_{m}(z, w)=\langle z, w\rangle^{m} / m$ !, and the FarautKorányi formula (75) holds if we take $(\nu)_{m}:=\nu^{m}$. Thus the flat case resembles the rank 1 situation.

\section{Existence of the ASymptotic EXPANSion}

Throughout the rest of this paper, we will be dealing only with the Toeplitz calculus $\mathcal{T}^{\mathbf{C}}$ on a complex bounded symmetric domain $D^{\mathbf{C}}$, and the real Toeplitz calculus $\mathcal{T}$ on the corresponding real domain $D$. Recall that these are given by

$$
\begin{aligned}
\mathcal{T}_{F}^{\mathbf{C}} & =\int_{D^{\mathbf{C}}} F(w) \mathcal{T}_{w}^{\mathbf{C}} d \mu_{0}^{\mathbf{C}}(w), \\
\mathcal{T}_{f} & =\int_{D} f(\zeta) \mathcal{T}_{\zeta} d \mu_{0}(\zeta),
\end{aligned}
$$

where $\mathcal{T}_{w}^{\mathbf{C}}$ and $\mathcal{T}_{\zeta}$ are operators on $H_{\nu}^{2}\left(D^{\mathbf{C}}\right)$ and elements of $H_{\nu}^{2}\left(D^{\mathbf{C}}\right)$, respectively, determined uniquely by

$$
\begin{aligned}
& \mathcal{T}_{g(w)}^{\mathbf{C}}=U_{g}^{(\nu)} \mathcal{T}_{w}^{\mathbf{C}} U_{g}^{(\nu) *}, \quad \forall g \in G^{\mathbf{C}}, \\
& \mathcal{T}_{g(\zeta)}=U_{g}^{(\nu)} \mathcal{T}_{\zeta}, \quad \forall g \in G,
\end{aligned}
$$

and

$$
\begin{aligned}
\mathcal{T}_{0}^{\mathrm{C}} & =\langle\cdot, \mathbf{1}\rangle \mathbf{1}, \\
\mathcal{T}_{0} & =\mathbf{1},
\end{aligned}
$$


respectively. Moreover

$$
\begin{aligned}
d \mu_{0}^{\mathbf{C}}(w) & =c_{\nu}^{\mathbf{C}} h(w, w)^{-p} d w, \\
d \mu_{0}(x) & =c_{\nu} h(x, x)^{-p / 2} d x
\end{aligned}
$$

denote the $G^{\mathbf{C}}$-invariant measure on $D^{\mathbf{C}}$ and the $G$-invariant measure on $D$, respectively, normalized so that $\mathcal{T}_{\mathbf{1}}^{\mathbf{C}}$ is the identity operator and $\mathcal{T}_{\mathbf{1}}=I$. (Thus $d \mu_{0}^{\mathbf{C}}$ and $d \mu_{0}$ also depend on $\nu$, though this is not reflected in the notation.)

For the complex Berezin transform $B_{\nu}^{\mathbf{C}}=\mathcal{T}^{\mathbf{C} *} \mathcal{T}^{\mathrm{C}}$, the following proposition is well-known; the proof for the real case is quite analogous, but we include both here for the sake of completeness.

Proposition 1. Let $F \in C^{\infty}\left(D^{\mathbf{C}}\right)$ and $f \in C^{\infty}(D)$ be such that $B_{\nu}^{\mathbf{C}} F$ and $B_{\nu} f$, respectively, are defined for some $\nu=\nu_{0}$. Then they exist also for all $\nu>\nu_{0}$, and as $\nu \rightarrow+\infty$, they have asymptotic expansions ${ }^{1}$

$$
\begin{aligned}
B_{\nu}^{\mathbf{C}} F(z) & \approx \sum_{n=0}^{\infty} \nu^{-n} Q_{n} F(z), \\
B_{\nu} f(x) & \approx \sum_{n=0}^{\infty} \nu^{-n} R_{n} f(x),
\end{aligned}
$$

for some $G^{\mathbf{C}}$-invariant linear differential operators $Q_{n}$ on $D^{\mathbf{C}}$ and $G$-invariant linear differential operators $R_{n}$ on $D$, respectively; further, $Q_{0}$ and $R_{0}$ are the identity operators.

Proof. From (78) and (79) one computes that

$$
\begin{aligned}
\mathcal{T}_{w}^{\mathbf{C}} & =h(w, w)^{\nu}\left\langle\cdot, h(\cdot, w)^{-\nu}\right\rangle h(\cdot, w)^{-\nu}, \\
\mathcal{T}_{x} & =h(x, x)^{\nu / 2} h(\cdot, x)^{-\nu} .
\end{aligned}
$$

The Berezin transforms are thus given by

$$
\begin{aligned}
B_{\nu}^{\mathbf{C}} F(z) & =\int_{D^{\mathbf{C}}} F(w) \operatorname{tr}\left(\mathcal{T}_{w}^{\mathbf{C}} \mathcal{T}_{z}^{\mathbf{C} *}\right) d \mu_{0}^{\mathbf{C}}(w) \\
& =\int_{D^{\mathbf{C}}} F(w) \frac{h(w, w)^{\nu} h(z, z)^{\nu}}{|h(z, w)|^{2 \nu}} d \mu_{0}^{\mathbf{C}}(w) \\
& =c_{\nu}^{\mathbf{C}} \int_{D^{\mathbf{C}}} F(w) \frac{h(z, z)^{\nu}}{|h(z, w)|^{2 \nu}} h(w, w)^{\nu-p} d w \\
B_{\nu} f(x) & =\int_{D} f(\zeta)\left\langle\mathcal{T}_{\zeta}, \mathcal{T}_{x}\right\rangle_{\nu} d \mu_{0}(\zeta) \\
& =\int_{D} f(\zeta) \frac{h(x, x)^{\nu / 2} h(\zeta, \zeta)^{\nu / 2}}{h(x, \zeta)^{\nu}} d \mu_{0}(\zeta) \\
& =c_{\nu} \int_{D} f(\zeta) \frac{h(x, x)^{\nu / 2}}{h(x, \zeta)^{\nu}} h(\zeta, \zeta)^{(\nu-p) / 2} d \zeta .
\end{aligned}
$$

We need to prove that these have asymptotic expansions (81) as $\nu \rightarrow+\infty$. Since everything is $G^{\mathbf{C}}$ - respectively $G$-invariant, it is enough to prove this for $z=0$

\footnotetext{
${ }^{1}$ Sometimes we will write simply "=" instead of " $\approx "$ in the sequel.
} 
and $x=0$, respectively. Since $h(\cdot, 0)=1$, we thus need to find the behaviour as $\nu \rightarrow+\infty$ of the integrals

$$
\begin{aligned}
B_{\nu}^{\mathbf{C}} F(0) & =c_{\nu}^{\mathbf{C}} \int_{D^{\mathbf{C}}} F(w) h(w, w)^{\nu-p} d w, \\
B_{\nu} f(0) & =c_{\nu} \int_{D} f(\zeta) h(\zeta, \zeta)^{(\nu-p) / 2} d \zeta .
\end{aligned}
$$

To this end, let us recall the familiar stationary phase method for the asymptotic expansions as $\nu \rightarrow+\infty$ of integrals of the form

$$
\mathcal{J}(\nu)=\int_{\Omega} f(x) e^{\nu S(x)} d x
$$

with smooth complex-valued functions $f, S$ on a domain $\Omega \subset \mathbf{R}^{N}$. Namely, assume that the following hypotheses are fulfilled:

- $f$ is compactly supported;

- $\operatorname{Re} S(x) \leq 0$, with equality if and only if $x=x_{0}$;

- $x_{0}$ is a critical point of $S$, i.e. $S^{\prime}\left(x_{0}\right)=0$; and

- this critical point is nondegenerate, i.e. $\operatorname{det} S^{\prime \prime}\left(x_{0}\right) \neq 0$.

Then as $\nu \rightarrow+\infty$,

$$
\mathcal{J}(\nu) \approx \nu^{-N / 2} \sum_{n=0}^{\infty} \nu^{-n} \mathcal{J}_{n} f\left(x_{0}\right),
$$

where $\mathcal{J}_{n}$ are some linear differential operators whose coefficients involve only $S$ and its derivatives; in particular,

$$
\mathcal{J}_{0} f\left(x_{0}\right)=(2 \pi)^{N / 2}\left|\operatorname{det} S^{\prime \prime}\left(x_{0}\right)\right|^{-1 / 2} f\left(x_{0}\right) .
$$

See, for instance, [15], Section 7.7. Further, the hypothesis of compact support of $f$ can be replaced by the two requirements that

- the integral $\mathcal{J}(\nu)$ exists for some $\nu=\nu_{0}$; and

- $\operatorname{Re} S$ is bounded away from zero at the boundary (and, if $\Omega$ is unbounded, at infinity), in the sense that

$$
x_{n} \in \Omega, \quad \operatorname{Re} S\left(x_{n}\right) \rightarrow 0 \Longrightarrow x_{n} \rightarrow x_{0} .
$$

Let us now apply this to the integrals (84). The phase function $S$ is in both cases given by

$$
S(z)=\log h(z, z)
$$

(The $S$ for the real case is just the restriction to $D$ of the $S$ from the complex case.) Thus $S$ is real-valued, and from $(70)$ it is immediate that it has a strict global maximum $S(0)=0$ at the origin and tends to $-\infty$ at the boundary. Thus all the hypothesis of the stationary phase method are satisfied, and so

$$
\begin{aligned}
& \int_{D^{\mathbf{C}}} F(w) h(w, w)^{\nu-p} d w \approx \nu^{-d} \sum_{n=0}^{\infty} \nu^{-n} \mathcal{J}_{n}^{\mathbf{C}} F(0), \\
& \int_{D} f(\zeta) h(\zeta, \zeta)^{(\nu-p) / 2} d \zeta \approx \nu^{-d / 2} \sum_{n=0}^{\infty} \nu^{-n} \mathcal{J}_{n} f(0),
\end{aligned}
$$

for some differential operators $\mathcal{J}_{n}^{\mathbf{C}}$ and $\mathcal{J}_{n}$ on $Z^{\mathbf{C}}$ and $Z$, respectively, with $\mathcal{J}_{0}^{\mathbf{C}} F(0)=$ $s_{0}^{\mathbf{C}} F(0)$ and $\mathcal{J}_{0} f(0)=s_{0} f(0)$ for some nonzero numbers $s_{0}^{\mathbf{C}}$ and $s_{0}$. Applying this, 
in particular, to $F=\mathbf{1}$ and $f=\mathbf{1}$, we get in view of the normalizations $B_{\nu}^{\mathrm{C}} \mathbf{1}=\mathbf{1}$ and $B_{\nu} \mathbf{1}=\mathbf{1}$

$$
\begin{aligned}
& 1 / c_{\nu}^{\mathbf{C}} \approx \nu^{-d} \sum_{n=0}^{\infty} \nu^{-n} \mathcal{J}_{n}^{\mathbf{C}} \mathbf{1}(0), \\
& 1 / c_{\nu} \approx \nu^{-d / 2} \sum_{n=0}^{\infty} \nu^{-n} \mathcal{J}_{n} \mathbf{1}(0),
\end{aligned}
$$

with $\mathcal{J}_{0}^{\mathbf{C}} \mathbf{1}(0)=s_{0}^{\mathbf{C}}$ and $\mathcal{J}_{0} \mathbf{1}(0)=s_{0}$. Combining (85) and (86), the desired asymptotics for the integrals (84) follows, completing the proof of the proposition.

Recall now that the "star restriction" operator corresponding to $\mathcal{T}^{\mathbf{C}}$ and $\mathcal{T}$ has been (so far, formally) defined by

$$
\mathcal{T}_{\# F}=\mathcal{T}_{F}^{\mathbf{C}} I, \quad F \in C^{\infty}\left(D^{\mathbf{C}}\right),
$$

where $I(z)=h\left(z, z^{\#}\right)^{-\nu / 2}$. Applying $\mathcal{T}^{*}$ to both sides yields

$$
B_{\nu} \# F=\mathcal{T}^{*} \mathcal{T}_{F}^{\mathbf{C}} I
$$

We have seen in the last proposition that $B_{\nu}$ has the asymptotic expansion (81) as $\nu \rightarrow+\infty$. This prompts the following definition, giving a rigorous sense to the equation (87).

Definition 2. By the star restriction $\# F$ of $F \in C^{\infty}\left(D^{\mathbf{C}}\right)$ we mean the formal power series

$$
\# F(z)=\sum_{n=0}^{\infty} \nu^{-n} L_{n} F(z)
$$

in $\frac{1}{\nu}$, such that $\mathcal{T}^{*} \mathcal{T}_{F}^{\mathbf{C}} I$ has the asymptotic expansion

$$
\begin{aligned}
\mathcal{T}^{*} \mathcal{T}_{F}^{\mathrm{C}} I(x) & \approx\left(\sum_{n=0}^{\infty} \nu^{-n} R_{n}\right)\left(\sum_{n=0}^{\infty} \nu^{-n} L_{n}\right) F(x) \\
& \approx \sum_{n=0}^{\infty} \nu^{-n} \sum_{j=0}^{n} R_{j} L_{n-j} F(x)
\end{aligned}
$$

(obtained by multiplying the asymptotic expansion for $B_{\nu}$ and the one from (89)) as $\nu \rightarrow+\infty$, for each $x \in D$.

The main result of this section is the following.

Theorem 3. The formal power series (89) exists and is unique; further, the $L_{n}$ are $G$-invariant differential operators from $C^{\infty}\left(D^{\mathbf{C}}\right)$ into $C^{\infty}(D)$.

Proof. It is enough to show that $\mathcal{T}^{*} \mathcal{T}_{F}^{\mathbf{C}} I$ has an asymptotic expansion

$$
\mathcal{T}^{*} \mathcal{T}_{F}^{\mathbf{C}}(x) \approx \sum_{n=0}^{\infty} \nu^{-n} M_{n} F(x)
$$

as $\nu \rightarrow+\infty$, for some differential operators $M_{n}$. Indeed, since $R_{0}=I$, one can then define the $L_{n}$ recursively by

$$
L_{n}=M_{n}-\sum_{i=1}^{n} R_{i} L_{n-j}
$$


Further, since the left-hand side of (91) is $G$-invariant, so will be the operators $M_{n}$, and, hence (since $R_{j}$ are $G$-invariant) also $L_{n}$, and we will be done.

Owing to $G$-invariance, it suffices to establish (91) for $x=0$. Using the formulas (77) and (82), we have

$$
\begin{aligned}
\mathcal{T}^{*} \mathcal{T}_{F}^{\mathbf{C}} I(0) & =\left\langle\mathcal{T}_{F}^{\mathbf{C}} I, \mathcal{T}_{0}\right\rangle_{\nu}=\left\langle\mathcal{T}_{F}^{\mathbf{C}} I, \mathbf{1}\right\rangle_{\nu}=\int_{D^{\mathbf{C}}} F(w)\left\langle\mathcal{T}_{w}^{\mathbf{C}} I, \mathbf{1}\right\rangle_{\nu} d \mu_{0}^{\mathbf{C}}(w) \\
& =\int_{D^{\mathbf{C}}} F(w) h(w, w)^{\nu} I(w) \overline{\mathbf{1}(w)} d \mu_{0}^{\mathbf{C}}(w) \\
& =c_{\nu}^{\mathbf{C}} \int_{D^{\mathbf{C}}} F(w) h(w, w)^{\nu-p} h\left(w, w^{\#}\right)^{-\nu / 2} d w
\end{aligned}
$$

The integral is again of the form susceptible to the stationary phase method, this time with the complex-valued phase function

$$
S(z)=\log h(z, z)-\frac{1}{2} \log h\left(z, z^{\#}\right) .
$$

We claim that the hypotheses of the stationary phase method are again satisfied, namely, that $z=0$ is a nondegenerate critical point of $S$, and that $\operatorname{Re} S \rightarrow-\infty$ at the boundary. Indeed, the Schwarz inequality

$$
|K(z, w)| \leq K(z, z)^{1 / 2} K(w, w)^{1 / 2}
$$

for the Bergman kernel $K(z, w)=h(z, w)^{-p}$ implies that

$$
|h(z, w)| \geq h(z, z)^{1 / 2} h(w, w)^{1 / 2} .
$$

Taking $z=w^{\#}$, we get $\left|h\left(z, z^{\#}\right)\right| \geq h(z, z) \operatorname{since} h\left(z^{\#}, z^{\#}\right)=h(z, z)$. Consequently,

$$
\operatorname{Re} S(z)=\log \frac{h(z, z)}{\left|h\left(z, z^{\#}\right)\right|^{1 / 2}} \leq \frac{1}{2} \log h(z, z),
$$

implying, by (70), that $\operatorname{Re} S(z) \leq 0$ with equality only for $z=0$, and that $\operatorname{Re} S \rightarrow$ $-\infty$ at the boundary. It remains to show that $z=0$ is a nondegenerate critical point. To this end, let us look at the Taylor expansion of $h(z, w)$ at $z=w=0$ : by the Faraut-Korányi formula (75),

$$
h(z, w)=1-\langle z, w\rangle+O\left(\|z\|^{2}\|w\|^{2}\right) .
$$

Hence

$$
S(z)=-\langle z, z\rangle+\frac{1}{2}\left\langle z, z^{\#}\right\rangle+O\left(\|z\|^{4}\right) .
$$

It follows that $S^{\prime}(0)=0$, while

$$
\frac{\partial^{2} S(0)}{\partial \bar{z}_{j} \partial \bar{z}_{k}}=0, \quad \frac{\partial^{2} S(0)}{\partial z_{j} \partial \bar{z}_{k}}=-\delta_{j k}
$$

so that

$$
\operatorname{det}\left[\begin{array}{ll}
\partial \bar{\partial} S & \partial \bar{\partial} S \\
\partial \bar{\partial} S & \partial \partial S
\end{array}\right]=\operatorname{det}\left[\begin{array}{cc}
0 & -I \\
-I & *
\end{array}\right]=1
$$

so $S^{\prime \prime}(0)$ is nondegenerate. By the stationary phase method, we thus get

$$
\int_{D^{\mathbf{C}}} F(w) h(w, w)^{\nu-p} h\left(w, w^{\#}\right)^{-\nu / 2} d w \approx \nu^{-d} \sum_{n=0}^{\infty} \nu^{-n} \widetilde{\mathcal{J}}_{n} F(0),
$$


for some differential operators $\widetilde{\mathcal{J}}_{n}$ on $Z^{\mathrm{C}}$. Combining this with the asymptotic expansion (86) for $c_{\nu}^{\mathbf{C}}$ and feeding these into (92), the expansion (91) at $x=0$ follows, thus completing the proof of the theorem.

Remark 4. It is not difficult to see that, in fact, $\widetilde{\mathcal{J}}_{0} F(0)=s_{0}^{\mathbf{C}} F(0)$, so $M_{0} F(0)=$ $F(0)$ and $L_{0} F(0)=F(0)$; consequently, by $G$-invariance, the leading term $L_{0}$ in $(89)$ is again the identity operator.

The asymptotic expansion (89) sometimes converges, so that \#F exists as an honest function on $D$. In the next section we show that this is the case, for instance, whenever $F$ is anti-holomorphic; further, this fact can be used to extract additional information about the operators $L_{n}$.

\section{ON SOME $G$-INVARIANT OPERATORS}

Let $L^{\mathbf{R}}$ be a $G$-invariant linear differential operator on $D$. In view of the $G$ invariance, $L^{\mathbf{R}}$ is uniquely determined by the constant-coefficient linear differential operator $\left(L^{\mathbf{R}}\right)_{0}$ obtained upon freezing the coefficients of $L^{\mathbf{R}}$ at the origin: indeed, from

$$
L^{\mathbf{R}} f(0)=\left(L^{\mathbf{R}}\right)_{0} f(0) \quad \forall f \in C^{\infty}(D)
$$

we obtain, replacing $f$ by $f \circ g_{x}$ for some $g_{x} \in G$ sending 0 into $x \in D$,

$$
L^{\mathbf{R}} f(x)=\left(L^{\mathbf{R}}\right)_{0}\left(f \circ g_{x}\right)(0) \quad \forall f .
$$

Further, the operator $\left(L^{\mathbf{R}}\right)_{0}$ must satisfy $\left(L^{\mathbf{R}}\right)_{0} f(0)=\left(L^{\mathbf{R}}\right)_{0}(f \circ k)(0)$ for any $k \in G$ fixing the origin, i.e. for any $k \in K$.

Being a constant coefficient linear differential operator, the operator $\left(L^{\mathbf{R}}\right)_{0}$ is of the form $l(\nabla)$, for some polynomial $l$ on $Z$. The $K$-invariance of $\left(L^{\mathbf{R}}\right)_{0}$ means that $l$ is also $K$-invariant, i.e. $l(x)=l(k x) \forall k \in K$. We define the complexification $L^{\mathbf{C}}$ of $L^{\mathbf{R}}$ to be the $G$-invariant operator from functions on $D^{\mathbf{C}}$ into functions on $D$ for which

$$
L^{\mathbf{C}} F(0):=l(\partial) F(0)
$$

In other words, we replace in $\left(L^{\mathbf{R}}\right)_{0}$ the real derivatives $\nabla$ by the holomorphic derivatives $\partial$. For $x \in D, L^{\mathbf{C}} F(x)$ is given by

$$
L^{\mathbf{C}} F(x)=l(\partial)(F \circ g)(0)
$$

for any $g \in G$ sending 0 into $x$; owing to the $K$-invariance of $l$, the right-hand side does not depend on the choice of $g$ (since any two such $g$ differ by an element of $K$ ).

Proposition 5. Let $L$ be a $G$-invariant differential operator from $C^{\infty}\left(D^{\mathbf{C}}\right)$ into $C^{\infty}(D)$. Then the following are equivalent:

(a) $L=L^{\mathbf{C}}$ is the complexification of some G-invariant differential operator $L^{\mathbf{R}}$ on $D$;

(b) L has the "factorization-property"

$$
L(\bar{H} F)=\bar{H} \cdot L F
$$

for any holomorphic function $H$ on $D^{\mathbf{C}}$.

(More precisely, on the last line we should write $L(\bar{H} F)=\overline{\rho H} \cdot L F$, where $\rho$ denotes the restriction map from $D^{\mathbf{C}}$ to $D$.) 
Proof. That (a) implies (b) is an immediate consequence of the definition (93), since $\partial(\bar{H} F)=\bar{H} \partial F$ for holomorphic $H$. To prove the converse, it is enough to show that $L F(0)=(l(\partial) F)(0)$ for some (necessarily $K$-invariant) polynomial $l$ on $Z$, since then $L=L^{\mathbf{C}}$ for the (uniquely determined) $G$-invariant operator $L^{\mathbf{R}}$ on $D$ satisfying $\left(L^{\mathbf{R}}\right)_{0}=l(\nabla)$. Now since $L$ is a differential operator,

$$
L F(0)=\sum_{\alpha, \beta} a_{\alpha \beta} \partial^{\alpha} \bar{\partial}^{\beta} F(0),
$$

where the summation extends over all multiindices $\alpha, \beta$, and $a_{\alpha \beta}$ are some complex numbers (only finitely many of which are nonzero). Thus if $F(z)=z^{A} \bar{z}^{B}$ and $H(z)=z^{C}$ for some multiindices $A, B$ and $C$, where $|C| \geq 1$, then

$$
(\bar{H} \cdot L F)(0)=\overline{H(0)} L F(0)=0,
$$

while

$$
L(\bar{H} F)(0)=\sum_{\alpha, \beta} a_{\alpha \beta} \partial^{\alpha} \bar{\partial}^{\beta}\left(z^{A} \bar{z}^{B+C}\right)(0)=A !(B+C) ! a_{A, B+C} .
$$

It follows that $a_{A, B}=0$ whenever $|B| \geq 1$. Consequently, setting $l(x)=\sum_{\alpha} a_{\alpha 0} x^{\alpha}$, we have

$$
L F(0)=\sum_{\alpha} a_{\alpha 0} \partial^{\alpha} F(0)=l(\partial) F(0)
$$

We now show that the star-restriction operator \# and, hence, also the operators $L_{m}$ from its asymptotic expansion (89), are of the type described in the last proposition.

Proposition 6. For $H$ holomorphic on $D^{\mathbf{C}}$ and any $F$,

$$
\#(\bar{H} F)=\bar{H} \cdot \# F \text {. }
$$

Proof. We claim that for any $f$ on $D$,

$$
\mathcal{T}_{\bar{H}}^{\mathbf{C}} \mathcal{T}_{f}=\mathcal{T}_{\bar{H} f}
$$

To see this, let $K_{z}$ temporarily denote the function $K^{(\nu)}(\cdot, z)=h(\cdot, z)^{-\nu}$. Then for any $z \in D^{\mathbf{C}}$, we have

$$
\begin{aligned}
\left(\mathcal{T}_{\bar{H}}^{\mathbf{C}} \mathcal{T}_{f}\right)(z) & =\int_{D^{\mathbf{C}}} \overline{H(w)}\left(\mathcal{T}_{w}^{\mathbf{C}} \mathcal{T}_{f}\right)(z) d \mu_{0}^{\mathbf{C}}(w) \\
& =\int_{D^{\mathbf{C}}} \overline{H(w)} h(w, w)^{\nu}\left\langle\mathcal{T}_{f}, K_{w}\right\rangle_{\nu} K_{w}(z) d \mu_{0}^{\mathbf{C}}(w) \\
& =\int_{D^{\mathbf{C}}} \overline{H(w)} \mathcal{T}_{f}(w) K^{(\nu)}(z, w) d \mu_{\nu}^{\mathbf{C}}(w) \\
& =\int_{D^{\mathbf{C}}} \int_{D} \overline{H(w)} f(\zeta) \mathcal{T}_{\zeta}(w) K^{(\nu)}(z, w) d \mu_{0}(\zeta) d \mu_{\nu}^{\mathbf{C}}(w) \\
& =\int_{D^{\mathbf{C}}} \int_{D} \overline{H(w)} f(\zeta) h(\zeta, \zeta)^{\nu / 2} K^{(\nu)}(w, \zeta) K^{(\nu)}(z, w) d \mu_{0}(\zeta) d \mu_{\nu}^{\mathbf{C}}(w) \\
& =\int_{D} f(\zeta) h(\zeta, \zeta)^{\nu / 2} \int_{D^{\mathbf{C}}} \overline{H(w)} K^{(\nu)}(w, \zeta) K^{(\nu)}(z, w) d \mu_{\nu}^{\mathbf{C}}(w) d \mu_{0}(\zeta) \\
& =\int_{D} f(\zeta) h(\zeta, \zeta)^{\nu / 2}\left\langle K_{\zeta}, H K_{z}\right\rangle_{\nu} d \mu_{0}(\zeta)
\end{aligned}
$$




$$
\begin{aligned}
& =\int_{D} f(\zeta) h(\zeta, \zeta)^{\nu / 2} \overline{H(\zeta) K_{z}(\zeta)} d \mu_{0}(\zeta) \\
& =\int_{D} f(\zeta) \overline{H(\zeta)} \mathcal{T}_{\zeta}(z) d \mu_{0}(\zeta)=\mathcal{T}_{\bar{H} f}(z)
\end{aligned}
$$

using (77), (82), (80), (1), Fubini's theorem, the reproducing property of $K_{\zeta}$, and (82) and (77) again. Next, we claim that

$$
\mathcal{T}_{\bar{H} F}^{\mathbf{C}}=\mathcal{T}_{\bar{H}}^{\mathbf{C}} \mathcal{T}_{F}^{\mathbf{C}}
$$

Indeed, for any $G \in \mathcal{H}^{\nu}$,

$$
\begin{aligned}
\left(\mathcal{T}_{\bar{H}}^{\mathbf{C}} \mathcal{T}_{F}^{\mathbf{C}} G\right)(z) & =\int_{D^{\mathbf{C}}} \int_{D^{\mathbf{C}}} \overline{H(y)} F(w)\left(\mathcal{T}_{t}^{\mathbf{C}} \mathcal{T}_{w}^{\mathbf{C}} G\right)(z) d \mu_{0}^{\mathbf{C}}(w) d \mu_{0}^{\mathbf{C}}(y) \\
& =\int_{D^{\mathbf{C}}} \int_{D^{\mathbf{C}}} \overline{H(y)} F(w)\left\langle G, K_{w}\right\rangle_{\nu}\left\langle K_{w}, K_{y}\right\rangle_{\nu} K_{y}(z) d \mu_{\nu}^{\mathbf{C}}(w) d \mu_{\nu}^{\mathbf{C}}(y) \\
& =\int_{D^{\mathbf{C}}} F(w)\left\langle G, K_{w}\right\rangle_{\nu} \overline{\int_{D^{\mathbf{C}}} H(y) K_{z}(y) \overline{K_{w}(y)} d \mu_{\nu}^{\mathbf{C}}(y)} d \mu_{\nu}^{\mathbf{C}}(w) \\
& =\int_{D^{\mathbf{C}}} F(w)\left\langle G, K_{w}\right\rangle \overline{H(w) K_{z}(w)} d \mu_{\nu}^{\mathbf{C}}(w) \\
& =\int_{D^{\mathbf{C}}}(\bar{H} F)(w)\left\langle G, K_{w}\right\rangle K_{w}(z) d \mu_{\nu}^{\mathbf{C}}(w) \\
& =\int_{D^{\mathbf{C}}}(\bar{H} F)(w)\left(\mathcal{T}_{w}^{\mathbf{C}} G\right)(z) d \mu_{0}^{\mathbf{C}}(w)=\left(\mathcal{T}_{\bar{H} F}^{\mathbf{C}} G\right)(z) .
\end{aligned}
$$

Now by (87), (95) and (94)

$$
\mathcal{T}_{\#(\bar{H} F)}=\mathcal{T}_{\bar{H} F}^{\mathbf{C}} I=\mathcal{T}_{\bar{H}}^{\mathbf{C}} \mathcal{T}_{F}^{\mathbf{C}} I=\mathcal{T}_{\bar{H}}^{\mathbf{C}} \mathcal{T}_{\# F}=\mathcal{T}_{\bar{H}(\# F)},
$$

so $\#(\bar{H} F)=\bar{H}(\# F)$.

Corollary 7. We have

$$
\# F=\sum_{n=0}^{\infty} \nu^{-n} L_{n}^{\mathbf{C}} F,
$$

where $L_{n}^{\mathbf{C}}$ are the complexifications of some $G$-invariant differential operators $L_{n}^{\mathbf{R}}$ on $D$.

Proof. Combine the last two propositions with Theorem 3.

Remark 8. A somewhat more expedient proof of (94) and (95) can be given by observing first that

$$
\mathcal{T}_{F}^{\mathbf{C}} \phi=P_{\nu}(F \phi),
$$

where $P_{\nu}: L^{2}\left(D^{\mathbf{C}}, d \mu_{\nu}^{\mathbf{C}}\right) \rightarrow \mathcal{H}^{\nu}\left(D^{\mathbf{C}}\right)$ is the orthogonal projection. (This is, in fact, the usual definition of Toeplitz operators.) Indeed, for any $\phi, \psi \in \mathcal{H}^{\nu}$,

$$
\begin{aligned}
\left\langle\mathcal{T}_{F}^{\mathbf{C}} \phi, \psi\right\rangle_{\nu} & =\int_{D^{\mathbf{C}}} F(z)\left\langle\mathcal{T}_{z}^{\mathbf{C}} \phi, \psi\right\rangle d \mu_{0}^{\mathbf{C}}(z) \\
& =\int_{D^{\mathbf{C}}} F(z) h(z, z)^{\nu}\left\langle\phi, K_{z}\right\rangle\left\langle K_{z}, \psi\right\rangle d \mu_{0}(z) \quad \text { by (82) } \\
& =\int_{D^{\mathbf{C}}} F(z) \phi(z) \overline{\psi(z)} h(z, z)^{\nu} d \mu_{0}(z)=\langle F \phi, \psi\rangle_{\nu},
\end{aligned}
$$


and (97) follows. The formula (95) then follows from the straightforward fact that $\mathcal{T}_{F}^{\mathbf{C}} \mathcal{T}_{H}^{\mathbf{C}}=\mathcal{T}_{F H}^{\mathbf{C}}\left(\right.$ and, hence, $\mathcal{T}_{\bar{H}}^{\mathbf{C}} \mathcal{T}_{F}^{\mathbf{C}}=\mathcal{T}_{\bar{H}}^{\mathbf{C}}$ ) whenever $H$ is holomorphic; similarly, (94) is immediate from the well-known relation

$$
\mathcal{T}_{\bar{H}}^{\mathbf{C}} K_{z}=\overline{H(z)} K_{z}
$$

and the formula $\mathcal{T}_{\zeta}=K_{\zeta} /\left\|K_{\zeta}\right\|$.

In view of Proposition 6, the star-restriction operator \# can be described by using its action on holomorphic functions $H$ on $D^{\mathbf{C}}$. In this case, we can describe $\# H$ rather neatly.

Proposition 9. For $H$ holomorphic on $D^{\mathbf{C}}$,

$$
B_{\nu} \# H=H \text {. }
$$

That is,

$$
\# H=\left(B_{\nu}\right)^{-1} \rho H
$$

where $\left(B_{\nu}\right)^{-1}$ is the inverse of the formal power series (81):

$$
\left(B_{\nu}\right)^{-1}=I-\frac{R_{1}}{\nu}+\frac{R_{1}^{2}-R_{2}}{\nu^{2}}+\ldots
$$

and $\rho$ stands, as before, for the restriction from $D^{\mathbf{C}}$ to $D$.

Proof. By (88) and (97) we have

$$
\begin{aligned}
B_{\nu} \# H(0) & =\left(\mathcal{T}^{*} \mathcal{T}_{H}^{\mathbf{C}} I\right)(0)=\left\langle\mathcal{T}_{H}^{\mathbf{C}} I, \mathcal{T}_{0}\right\rangle=\left\langle\mathcal{T}_{H}^{\mathbf{C}} I, \mathbf{1}\right\rangle \\
& =\langle H I, \mathbf{1}\rangle=H(0) I(0)=H(0) .
\end{aligned}
$$

By $G$-invariance, it follows that $B_{\nu} \# H=H$.

By the Harish-Chandra isomorphism, the operators $L_{n}^{\mathbf{R}}$ in (96) are uniquely determined by their eigenvalues $\widetilde{L_{n}^{\mathbf{R}}}(\lambda)$ on the conical functions $e^{\lambda, b}$ of $D$ :

$$
L_{n}^{\mathbf{R}} e^{\lambda, b}=\widetilde{L_{n}^{\mathbf{R}}}(\lambda) e^{\lambda, b}
$$

It can be shown that $e^{\lambda, b}$ are the restrictions to $D$ of certain holomorphic functions $E^{\lambda, b}$ on $D^{\mathbf{C}}$; cf. Lemma 2.3 in [26]. On the other hand, by (93) we then have for any holomorphic function $H$ on $D^{\mathbf{C}}$

$$
L_{n}^{\mathbf{C}} H(0)=l_{n}(\partial) H(0)=l_{n}(\nabla) H(0)=L_{n}^{\mathbf{R}}(\rho H)(0),
$$

(where $\rho$ again stands for the operator of restriction to $D$ ). By $G$-invariance, it follows that

$$
\rho L_{n}^{\mathbf{C}} H=L_{n}^{\mathbf{R}}(\rho H)
$$

In particular,

and, consequently, since $e^{\lambda, b}(0)=1$,

$$
\rho L_{n}^{\mathbf{C}} E^{\lambda, b}=L_{n}^{\mathbf{R}} e^{\lambda, b}=\widetilde{L_{n}^{\mathbf{R}}}(\lambda) e^{\lambda, b}
$$

$$
\# E^{\lambda, b}(0)=\sum_{n=0}^{\infty} \nu^{-n} \widetilde{L_{n}^{\mathbf{R}}}(\lambda) .
$$

Applying Proposition 9 and recalling that $B_{\nu} e^{\lambda, b}=b^{\nu}(\lambda) e^{\lambda, b}$, we thus get

$$
\frac{1}{b^{\nu}(\lambda)}=\sum_{n=0}^{\infty} \nu^{-n} \widetilde{L_{n}^{\mathbf{R}}}(\lambda) \text {. }
$$


Using the formula (44), it is in principle possible to extract from here the expressions for $\widetilde{L_{n}^{\mathbf{R}}}(\lambda)$ and, hence, up to the Harish-Chandra isomorphism, the operators $L_{n}^{\mathbf{R}}$ and $L_{n}=L_{n}^{\mathbf{C}}$.

Remark 10. Heuristically, we can rewrite (99) as

$$
\#=\left(\left(B_{\nu}\right)^{-1}\right)^{\mathbf{C}},
$$

i.e. the star restriction is the complexification — in the sense of (93) — of the inverse of the real Berezin transform.

Example 11. For $D=\mathbf{C}^{d}$, i.e. the "product case" for the Euclidean $d$-space, we have by (66)

$$
\#=\left(\left(B_{\nu}\right)^{-1}\right)^{\mathbf{C}}=\left(e^{-\Delta / 4 \nu}\right)^{\mathbf{C}}
$$

that is,

$$
\#(f \otimes g)(z)=\left.e^{-\partial_{x} \bar{\partial}_{y} / \nu} f(x) g(y)\right|_{x=y=z},
$$

so

$$
L_{n}(f \otimes g)=(-1)^{n} \sum_{|\alpha|=n} \frac{1}{\alpha !} \partial^{\alpha} f \cdot \bar{\partial}^{\alpha} g .
$$

Similarly, for the real Euclidean $d$-space $D=\mathbf{R}^{d}$, by (48) again

$$
\#=\left(\left(B_{\nu}\right)^{-1}\right)^{\mathbf{C}}=\left(e^{-\Delta / 2 \nu}\right)^{\mathbf{C}}=e^{-\partial^{2} / 2 \nu}
$$

so that

$$
L_{2 n-1}=0, \quad L_{2 n}=\frac{(-1)^{n}}{2^{n}} \sum_{|\alpha|=n} \frac{1}{\alpha !} \partial^{2 \alpha} .
$$

(In both cases, the summation extends over all multiindices $\alpha$ of total degree $n$.)

For later use, we put down the following proposition.

Proposition 12. Let $H$ be holomorphic on $D^{\mathbf{C}}$. Then

$$
B_{\nu}(\rho H)(0)=\langle H, I\rangle_{\nu}
$$

where $I$ is as in (17).

Proof. From (84) and (80),

$$
\begin{aligned}
B_{\nu}(\rho H)(0) & =c_{\nu} \int_{D} H(\zeta) h(\zeta, \zeta)^{(\nu-p) / 2} d \zeta \\
& =\int_{D} H(\zeta) I(\zeta)^{-1} d \mu_{0}=\langle H, I\rangle_{\nu}
\end{aligned}
$$

by Proposition 3.4 in [3]. 


\section{AraZy-Ørsted FORMUla FOR REAL SYMMETRIC DOMAINS}

In this section we generalize the "spectral-theoretic" asymptotic expansion of the (complex) Berezin transform due to Arazy-Ørsted [1] to the case of real symmetric domains. The Arazy-Ørsted formula (30) (in the complex bounded case) expresses the Berezin transform $B_{\mathbf{R}, \nu}$ in terms of differential operators constructed from $K^{\mathbf{m}}(z, w)$. The asymptotic expansion of the flat Berezin transform follows of course from the explicit realization (66).

Now suppose that $D \subset Z$ is an irreducible real bounded symmetric domain. Let $X \subset X_{\mathbf{C}}$ be the Euclidean Jordan algebras associated with $D$ (cf.(39)), $K_{X}$ the subgroup of all elements in $K$ which map $X$ into itself, $L$ the subgroup of $K$ stabilizing the maximal tripotent $e$, and $L_{X}:=L \cap K_{X}$. For each $m=0,1,2, \ldots$, the vector space $\mathcal{P}^{m}(X)$ of (complex) polynomials on $X$ homogeneous of degree $m$ has the Peter-Weyl decomposition under $K_{X}$

$$
\mathcal{P}^{m}(X)=\sum_{|\mathbf{m}|=m} \mathcal{P}^{\mathbf{m}}(X)
$$

where $\mathbf{m}=\left(m_{1}, \ldots, m_{r}\right)$ ranges over all integer partitions $m_{1} \geq \cdots m_{r} \geq 0$ of length $r$ satisfying $|\mathbf{m}|:=m_{1}+\cdots+m_{r}=m$. Thus we have also the decomposition

$$
\mathcal{P}(X)=\sum_{\mathbf{m}} \mathcal{P}^{\mathbf{m}}(X)
$$

of the whole (complex) polynomial algebra $\mathcal{P}(X) \equiv \mathcal{P}\left(X^{\mathbf{C}}\right)$. It is known that [25]

$$
d_{\mathbf{m}}:=\operatorname{dim}_{\mathbf{C}} \mathcal{P}^{\mathbf{m}}(X)=\prod_{1 \leq i<j \leq r} \frac{m_{i}-m_{j}+\frac{j-i}{2} a}{\frac{j-i}{2} a} \frac{\left(\frac{j-i+1}{2} a\right)_{m_{i}-m_{j}}}{\left(\frac{j-i-1}{2} a+1\right)_{m_{i}-m_{j}}} .
$$

Furthermore, for each $\mathbf{m}, \mathcal{P}^{\mathbf{m}}(X)$ contains a unique $L_{X}$-invariant ("spherical") polynomial $\phi^{\mathbf{m}}$ normalized by $\phi^{\mathbf{m}}(e)=1$. Similarly, we have the analogous decomposition of $\mathcal{P}\left(X_{\mathbf{C}}\right) \equiv \mathcal{P}\left(X_{\mathbf{C}}^{\mathbf{C}}\right)$ under $K^{\mathbf{C}}$, labelled by signatures of length $r_{\mathbf{C}}$.

Consider a $G$-invariant linear differential operator $L$ on $D$. As we have seen in the preceding section, it is uniquely determined by its "freezing at the origin" $(L)_{0}$, which is a $K$-invariant constant coefficient differential operator, hence of the form $p(\nabla)$ for some $K$-invariant polynomial $p(x)$ on $Z$. By holomorphic continuation, we may view $p$ also as a holomorphic polynomial $p(z)$ on $Z^{\mathbf{C}}$, and therefore decompose it into its Peter-Weyl components with respect to $K^{\mathbf{C}}$. Since $p$ is $K$-invariant, only those signatures whose Peter-Weyl spaces contain a nonzero $K$-invariant polynomial actually occur; we will call such signatures even. ${ }^{2}$

It turns out that (with the exception of type (A); see below) all even signatures can be expressed by specifying a partition $\mathbf{m}=\left(m_{1}, \ldots, m_{r}\right)$ of length $r$, and for the associated $K^{\mathbf{C}}$-signature $\mathbf{m}_{\mathbf{C}}$ (of length $r_{\mathbf{C}}$ ) the corresponding $K$-invariant polynomial $\phi_{\mathbf{C}}^{\mathbf{m}}$ on $Z^{\mathbf{C}}$ is always unique if we require that $\phi_{\mathbf{C}}^{\mathbf{m}}(e)=1$, and is closely related to the spherical polynomial $\phi^{\mathbf{m}} \in \mathcal{P}^{\mathbf{m}}(X)$ discussed in the penultimate paragraph.

The above decomposition is then a finite sum

$$
p(z)=\sum_{\mathbf{m}} c_{\mathbf{m}} \phi_{\mathbf{C}}^{\mathbf{m}}(z), \quad(L)_{0}=\sum_{\mathbf{m}} c_{\mathbf{m}} \phi_{\mathbf{C}}^{\mathbf{m}}(\nabla),
$$

with some constants $c_{\mathbf{m}}$.

\footnotetext{
${ }^{2}$ The term "spherical" was used in [27] and [28].
} 
Definition 13. The Peter-Weyl components $L_{\mathbf{m}_{\mathbf{C}}}$ of the operator $L$ are the $G$ invariant differential operators on $D$ whose action at the origin is given by

$$
\left(L_{\mathbf{m}_{\mathbf{C}}}\right)_{0}:=c_{\mathbf{m}} \phi_{\mathbf{C}}^{\mathbf{m}}(\nabla) \text {. }
$$

All this easily extends, in an appropriate sense, also to $G$-invariant operators on $D$ which are not necessarily differential, but have an asymptotic expansion of some kind whose coefficients are differential operators of the above form. (The only difference is that the sum (102) will then be infinite in general.) In particular, this applies to the real Berezin transform $B_{\nu}$ with the asymptotic expansion (81). Thus we define

$$
\left(B_{\nu}\right)_{\mathbf{m}_{\mathrm{C}}}:=\sum_{n=0}^{\infty} \nu^{-n}\left(R_{n}\right)_{\mathbf{m}_{\mathbf{C}}},
$$

where $R_{n}$ are the operators from (81).

For the complex case $D_{\mathbf{R}}^{\mathbf{C}}=D \times \bar{D}$, with $D$ an irreducible complex bounded symmetric domain, the signatures of $K_{\mathbf{R}}^{\mathbf{C}}$ are the ordered pairs $(\mathbf{m}, \mathbf{n})$ of signatures of $K$, the even ones are those with $\mathbf{m}=\mathbf{n}$, and the polynomials $\phi_{\mathbf{C}}^{\mathbf{m}}$ are the FarautKorányi kernels $K^{\mathbf{m}}(z, w)$ divided by $K^{\mathbf{m}}(e, e)$. In this setting, Arazy and Ørsted [1] showed that

$$
\left(B_{\nu}\right)_{(\mathbf{m}, \mathbf{m})}=\frac{K^{\mathbf{m}}(\partial, \partial)}{(\nu)_{\mathbf{m}}}
$$

for the Pochhammer symbols (76). Our next result asserts that there is an analogue of the Arazy-Ørsted formula also in the general case.

Theorem 14. For domains of type (A), the formal power series (104) is the asymptotic expansion as $\nu \rightarrow+\infty$ of

$$
\left(B_{\nu}\right)_{\mathbf{m}}=\frac{d_{\mathbf{m}}}{(d / r)_{\mathbf{m}}} \frac{\llbracket \nu \rrbracket_{\mathbf{m}}}{(\nu)_{\mathbf{m}}} \Phi^{\mathbf{m}}
$$

where $\mathbf{m}$ is any partition such that $|\mathbf{m}|$ is even, $\Phi^{\mathbf{m}}$ is the $G$-invariant operator on $C^{\infty}(D)$ whose action at the origin is given by

$$
\Phi^{\mathbf{m}} f(0)=\phi^{\mathbf{m}}(\nabla) f(0),
$$

and $\llbracket \nu \rrbracket_{\mathrm{m}}$ is defined by the formula (118) below.

For domains not of type (A), including the complex case, the formal power series (104) is the asymptotic expansion as $\nu \rightarrow+\infty$ of

$$
\left(B_{\nu}\right)_{\mathbf{m}_{\mathbf{C}}}=\frac{d_{\mathbf{m}}}{\left(2 r / r_{\mathbf{C}}\right)^{2|m|}} \frac{\Phi_{\mathbf{C}}^{\mathbf{m}}}{\left(\frac{d_{X}}{r}\right)_{\mathbf{m}}\left(\nu_{\mathbf{R}}+\frac{d_{X}-d_{Y}}{2 r}\right)_{\mathbf{m}}},
$$

where $\Phi_{\mathbf{C}}^{\mathbf{m}}$ is the $G$-invariant operator on $C^{\infty}(D)$ whose action at the origin is given by

and $\nu_{\mathbf{R}}$ is as in (45).

$$
\Phi_{\mathbf{C}}^{\mathbf{m}} f(0):=\phi_{\mathbf{C}}^{\mathbf{m}}(\nabla) f(0),
$$

Before proceeding to the proof, we first describe the "even" signatures $\mathbf{m}_{\mathbf{C}}$, associated with a partition $\mathbf{m}$, and the corresponding normalized $K$-invariant polynomial $\phi_{\mathbf{C}}^{\mathbf{m}}$ in more detail, using the fine structure of symmetric domains explained in Section 2.

For the domains of type (A), we have $\mathbf{m}_{\mathbf{C}}=\mathbf{m}$ and $\phi_{\mathbf{C}}^{\mathbf{m}}=\phi^{\mathbf{m}}$, but in this case the group $G$ is not connected and semisimple, more precisely it is the semidirect 
product of the connected component $(G)^{0}$ of the identity and the two-element group generated by the reflection $z \mapsto-z$; similarly the group $K$ is the semidirect product of the stabilizer $L$ of $e$ in $K^{\mathbf{C}}$ and the same two-element subgroup. Accordingly, a $K^{\mathbf{C}}$-signature $\mathbf{m}$ is even if and only if $|\mathbf{m}|:=m_{1}+\cdots+m_{r}$ is even.

For all other types, note first of all that by holomorphy, any polynomial on $Z^{\mathbf{C}}$ is uniquely determined by its restriction to $Z$; and by Chevalley's theorem (cf. [27], Proposition 6.2) and the "polar decomposition" [11, Section VI.2]

$$
Z=K \cdot \sum_{j=1}^{r} \mathbf{R} e_{j}
$$

(where $e_{1}, \ldots, e_{r}$ is any Jordan frame in $Z$ with $e_{1}+\cdots+e_{r}=e$ ), any polynomial in some $\mathcal{P}^{\mathbf{m}_{\mathbf{C}}}\left(Z^{\mathbf{C}}\right)$ has its values on $Z$ uniquely determined by its restriction to $\sum_{j=1}^{r} \mathbf{R} e_{j} \subset X$. Therefore, to describe the polynomials $\phi_{\mathbf{C}}^{\mathbf{m}} \in \mathcal{P}^{\mathbf{m}_{\mathbf{C}}}\left(Z^{\mathbf{C}}\right)$ it is enough to give their restrictions on $X$.

For type (D/B) we are likewise considering invariance under the full, not necessarily connected, group $K$. Hence the results of [27], [28] imply that a $K^{\mathbf{C}_{\text {-signature }}}$ (of length $r_{\mathbf{C}}=r$ ) is even if and only if it has the form

$$
\left(2 m_{1}, 2 m_{2}, \ldots, 2 m_{r}\right)=2 \mathbf{m}=: \mathbf{m}_{\mathbf{C}},
$$

where $\mathbf{m}$ is any signature of length $r$, and moreover

$$
\phi_{\mathbf{C}}^{\mathbf{m}}(x)=\phi^{\mathbf{m}}\left(x^{2}\right)
$$

for all $x \in X \subset X_{\mathbf{C}}$. (More precisely: for a $K^{\mathbf{C}}$-signature $\mathbf{n}, \mathcal{P}^{\mathbf{n}}\left(Z^{\mathbf{C}}\right)$ contains an $L$-invariant polynomial if and only if $\mathbf{n}=2 \mathbf{m}+\epsilon \cdot(1, \ldots, 1)$, where $\epsilon \in\{0,1\}$; and containing even a $K$-invariant polynomial forces $\epsilon=0$.) Since $X_{\mathbf{C}}$ is irreducible, with $r_{\mathbf{C}}=r$ and $a_{\mathbf{C}}=2 a$, we obtain [28, Proposition 3.5]

$$
\begin{aligned}
\frac{\left\|\phi_{\mathbf{C}}^{\mathbf{m}}\right\|_{F}^{2}}{\left\|\phi_{\mathbf{C}}^{\mathbf{m}}\right\|_{\nu}^{2}} & =\frac{\Gamma_{\mathbf{C}}(\nu+2 \mathbf{m})}{\Gamma_{\mathbf{C}}(\nu)} \\
& =\prod_{j=1}^{r} \frac{\Gamma\left(\nu+2 m_{j}-\frac{a_{\mathbf{C}}}{2}(j-1)\right)}{\Gamma\left(\nu-\frac{a_{\mathbf{C}}}{2}(j-1)\right)}=\prod_{j=1}^{r} \frac{\Gamma\left(\nu+2 m_{j}-a(j-1)\right)}{\Gamma(\nu-a(j-1))} \\
& =\prod_{j=1}^{r} \frac{2^{2 m_{j}} \Gamma\left(\frac{\nu}{2}+m_{j}-\frac{a}{2}(j-1)\right) \Gamma\left(\frac{\nu+1}{2}+m_{j}-\frac{a}{2}(j-1)\right)}{\Gamma\left(\frac{\nu}{2}-\frac{a}{2}(j-1)\right) \Gamma\left(\frac{\nu+1}{2}-\frac{a}{2}(j-1)\right)} \\
& =2^{2|m|} \frac{\Gamma\left(\frac{\nu}{2}+\mathbf{m}\right) \Gamma\left(\frac{\nu+1}{2}+\mathbf{m}\right)}{\Gamma\left(\frac{\nu}{2}\right) \Gamma\left(\frac{\nu+1}{2}\right)}=2^{2|m|}\left(\frac{\nu}{2}\right)_{\mathbf{m}}\left(\frac{\nu+1}{2}\right)_{\mathbf{m}}
\end{aligned}
$$

using the duplication formula for the ordinary $\Gamma$-function. For type $(\mathbf{C} / \mathbf{B C})$, the even $K^{\mathbf{C}}$-signatures (of length $r_{\mathbf{C}}=2 r$ ) can be written in the form

$$
\left(m_{1}, m_{1}, m_{2}, m_{2}, \ldots, m_{r}, m_{r}\right)=(\mathbf{m}, \mathbf{m})=: \mathbf{m}_{\mathbf{C}},
$$

where $\mathbf{m}$ is any signature of length $r$, and

$$
\phi_{\mathbf{C}}^{\mathbf{m}}(x)=\phi^{\mathbf{m}}\left(x^{2}\right)
$$

for all $x \in X \subset X_{\mathbf{C}}$. Since $X_{\mathbf{C}}$ is irreducible, with $r_{\mathbf{C}}=2 r$ and $a_{\mathbf{C}}=\frac{a}{2}$, we obtain [28, Proposition 3.5]

$$
\frac{\left\|\phi_{\mathbf{C}}^{\mathbf{m}}\right\|_{F}^{2}}{\left\|\phi_{\mathbf{C}}^{\mathbf{m}}\right\|_{\nu}^{2}}=\frac{\Gamma_{\mathbf{C}}(\nu+(\mathbf{m}, \mathbf{m}))}{\Gamma_{\mathbf{C}}(\nu)}
$$




$$
\begin{aligned}
& =\prod_{j=1}^{r} \frac{\Gamma\left(\nu+m_{j}-\frac{a_{\mathrm{C}}}{2}(2 j-2)\right) \Gamma\left(\nu+m_{j}-\frac{a_{\mathrm{C}}}{2}(2 j-1)\right)}{\Gamma\left(\nu-\frac{a_{\mathrm{C}}}{2}(2 j-2)\right) \Gamma\left(\nu-\frac{a_{\mathrm{C}}}{2}(2 j-1)\right)} \\
& =\prod_{j=1}^{r} \frac{\Gamma\left(\nu+m_{j}-\frac{a}{2}(j-1)\right) \Gamma\left(\nu+m_{j}-\frac{a}{2}(j-1)-\frac{a}{4}\right)}{\Gamma\left(\nu-\frac{a}{2}(j-1)\right) \Gamma\left(\nu-\frac{a}{2}(j-1)-\frac{a}{4}\right)} \\
& =\frac{\Gamma(\nu+\mathbf{m})}{\Gamma(\nu)} \frac{\Gamma\left(\nu+\mathbf{m}-\frac{a}{4}\right)}{\Gamma\left(\nu-\frac{a}{4}\right)}=(\nu)_{\mathbf{m}}\left(\nu-\frac{a}{4}\right)_{\mathbf{m}} .
\end{aligned}
$$

For the complex case, the even signatures for $K_{\mathbf{R}}^{\mathbf{C}}=K \times \bar{K}$ are of the form

$$
\left(m_{1}, m_{2}, \ldots, m_{r} ; m_{1}, m_{2}, \ldots, m_{r}\right)=(\mathbf{m}, \overline{\mathbf{m}})=: \mathbf{m}_{\mathbf{C}},
$$

where $\mathbf{m}$ is any signature of length $r$ and the bar denotes the "conjugate" second variable, and

$$
\phi_{\mathbf{C}}^{\mathbf{m}}(z, \bar{w})=\frac{K_{\mathbf{m}}(z, w)}{K_{\mathbf{m}}(e, e)}=\frac{\left(d_{X} / r\right)_{\mathbf{m}}}{d_{\mathbf{m}}} K^{\mathbf{m}}(z, w) .
$$

In particular, for $x \in X$ (identified with $x_{\mathbf{R}}:=(x, \bar{x}) \in X_{\mathbf{R}} \approx X$ ) we have

$$
\phi_{\mathbf{C}}^{\mathbf{m}}(x) \equiv \phi_{\mathbf{C}}^{\mathbf{m}}(x, \bar{x})=\frac{K^{\mathbf{m}}(x, x)}{K^{\mathbf{m}}(e, e)}=\frac{\left(d_{X} / r\right)_{\mathbf{m}}}{d_{\mathbf{m}}} K^{\mathbf{m}}(x, x)=\phi^{\mathbf{m}}\left(x^{2}\right) .
$$

Since $X_{\mathbf{C}}=X \times \bar{X}$ (with $r_{\mathbf{C}}=2 r$ and $a_{\mathbf{C}}=a$ ) is also of product type, we obtain [28, Lemma 3.1]

$$
\frac{\left\|\phi_{\mathbf{C}}^{\mathbf{m}}\right\|_{F}^{2}}{\left\|\phi_{\mathbf{C}}^{\mathbf{m}}\right\|_{\nu}^{2}}=\frac{\Gamma_{\mathbf{C}}(\nu+(\mathbf{m}, \overline{\mathbf{m}}))}{\Gamma_{\mathbf{C}}(\nu)}=\left[\frac{\Gamma(\nu+\mathbf{m})}{\Gamma(\nu)}\right]^{2}=(\nu)_{m}^{2} .
$$

Summarizing the three non-type (A) cases we see that

$$
\phi_{\mathbf{C}}^{\mathbf{m}}(x)=\phi^{\mathbf{m}}\left(x^{2}\right)
$$

and

$$
\frac{\left\|\phi_{\mathbf{C}}^{\mathbf{m}}\right\|_{F}^{2}}{\left\|\phi_{\mathbf{C}}^{\mathbf{m}}\right\|_{\nu}^{2}}=\left(\frac{2 r}{r_{\mathbf{C}}}\right)^{2|\mathbf{m}|}\left(\nu_{\mathbf{R}}\right)_{\mathbf{m}}\left(\nu_{\mathbf{R}}+\frac{d_{X}-d_{Y}}{2 r}\right)_{\mathbf{m}},
$$

where $\nu_{\mathbf{R}}$ is as in (45), i.e.

$$
\nu_{\mathbf{R}}=\frac{\nu r_{\mathbf{C}}}{2 r} .
$$

In fact, for complex type we have $\nu_{\mathbf{R}}=\nu$ and $d_{X}=d_{Y}$ since $Y=i X$. For type $(\mathbf{D} / \mathbf{B})$ we have $\nu_{\mathbf{R}}=\frac{\nu}{2}$ and $d_{X}-d_{Y}=r$. For type $(\mathbf{C} / \mathbf{B C})$ we have $\nu_{\mathbf{R}}=\nu$ and $\frac{d_{Y}-d_{X}}{2 r}=\frac{a}{4}$. This case is slightly subtle, since there exist two rank 1 domains in this class, for which we define $a:=\frac{a_{\mathbf{C}}}{2}$ (since $r_{\mathbf{C}}=2, a_{\mathbf{C}}$ is well-defined). One easily shows that the above relation holds also for the rank 1 cases.

Proof of Theorem 14. Consider the expansion

$$
I(z)=\sum_{\mathbf{m}} c_{\mathbf{m}}^{\nu} \phi_{\mathbf{C}}^{\mathbf{m}}(z)
$$

of the $K$-invariant holomorphic function $I$ on $D^{\mathbf{C}}$. Here $\mathbf{m}$ ranges over all partitions of length $r$, with $|\mathbf{m}|$ even for type (A) domains. Recalling the notation $f^{*}(z):=\overline{f\left(z^{\#}\right)}$, note that $\left\langle f^{*}, g^{*}\right\rangle_{F}=\langle g, f\rangle_{F}$ while, by the Hermitian symmetry 
of the Bergman kernels, $I^{*}(z)=\overline{K^{(\nu)}\left(z^{\#}, z\right)^{1 / 2}}=I(z)$. Thus for any holomorphic polynomial $H$ on $Z^{\mathrm{C}}$,

$$
\langle H, I\rangle_{\nu}=\left\langle I, H^{*}\right\rangle_{\nu}=\sum_{\mathbf{m}} c_{\mathbf{m}}^{\nu}\left\langle\phi_{\mathbf{C}}^{\mathbf{m}}, H^{*}\right\rangle_{\nu}=\sum_{\mathbf{m}} c_{\mathbf{m}}^{\nu} \frac{\left\|\phi_{\mathbf{C}}^{\mathbf{m}}\right\|_{\nu}^{2}}{\left\|\phi_{\mathbf{C}}^{\mathbf{m}}\right\|_{F}^{2}}\left\langle\phi_{\mathbf{C}}^{\mathbf{m}}, H^{*}\right\rangle_{F}
$$

since $\phi_{\mathbf{C}}^{\mathbf{m}}$ is pure of type $m_{\mathbf{C}}$. Putting

$$
\left(\left(B_{\nu}\right)_{\mathbf{m}}\right)_{0}=b_{\mathbf{m}}^{\nu} \phi_{\mathbf{C}}^{\mathbf{m}}(\nabla)
$$

Proposition 12 implies

$$
\begin{aligned}
\langle H, I\rangle_{\nu} & =B_{\nu}(\rho H)(0)=\sum_{\mathbf{m}} b_{\mathbf{m}}^{\nu} \phi_{\mathbf{C}}^{\mathbf{m}}(\nabla)(\rho H)(0) \\
& =\sum_{\mathbf{m}} b_{\mathbf{m}}^{\nu}\left(\phi_{\mathbf{C}}^{\mathbf{m}}(\partial) H\right)(0)=\sum_{\mathbf{m}} b_{\mathbf{m}}^{\nu}\left\langle\phi_{\mathbf{C}}^{\mathbf{m}}, H^{*}\right\rangle_{F} .
\end{aligned}
$$

It follows that

$$
b_{\mathbf{m}}^{\nu}=c_{\mathbf{m}}^{\nu} \frac{\left\|\phi_{\mathbf{C}}^{\mathbf{m}}\right\|_{\nu}^{2}}{\left\|\phi_{\mathbf{C}}^{\mathbf{m}}\right\|_{F}^{2}}
$$

Now for any $x \in D \cap X_{\mathbf{C}}$,

$$
I(x)=K^{(\nu)}(x, x)^{1 / 2}=h(x, x)^{-\nu / 2}=\Delta_{\mathbf{C}}\left(e-x^{2}\right)^{-\nu / 2},
$$

where $\Delta_{\mathbf{C}}$ is the Jordan algebra determinant of $X_{\mathbf{C}} \subset Z^{\mathbf{C}}$. It is known that $\Delta_{\mathbf{C}}=\Delta^{r_{\mathbf{C}} / r}$ on $X$, where $\Delta$ is the Jordan algebra determinant of $X \subset Z$. Thus

$$
I(x)=\Delta\left(e-x^{2}\right)^{-\nu_{\mathbf{R}}}=\sum_{\mathbf{m}} d_{\mathbf{m}} \frac{\left(\nu_{\mathbf{R}}\right)_{\mathbf{m}}}{\left(d_{X} / r\right)_{\mathbf{m}}} \phi^{\mathbf{m}}\left(x^{2}\right)
$$

for all $x \in D \cap X$, where we have applied the Faraut-Korányi binomial formula [11, Proposition XII.1.3] to $X$. For domains not of type (A), (110) therefore implies

$$
I(x)=\sum_{\mathbf{m}} d_{\mathbf{m}} \frac{\left(\nu_{\mathbf{R}}\right)_{\mathbf{m}}}{\left(d_{X} / r\right)_{\mathbf{m}}} \phi_{\mathbf{C}}^{\mathbf{m}}(x)
$$

for all $x \in D \cap X$. It follows that

$$
c_{\mathbf{m}}^{\nu}=d_{\mathbf{m}} \frac{\left(\nu_{\mathbf{R}}\right)_{\mathbf{m}}}{\left(d_{X} / r\right)_{\mathbf{m}}}
$$

for all partitions $\mathbf{m}$. Combining (115), (116) and (111) implies the assertion, namely

$$
b_{\mathbf{m}}^{\nu}=\frac{d_{\mathbf{m}}}{\left(d_{X} / r\right)_{\mathbf{m}}} \frac{\left(r_{\mathbf{C}} / 2 r\right)^{2 \mathbf{m}}}{\left(\nu_{\mathbf{R}}+\frac{d_{X}-d_{Y}}{2 r}\right)_{\mathbf{m}}} .
$$

For domains of type (A), we start with the decomposition

(118) $I(x)=\Delta\left(e-x^{2}\right)^{-\nu / 2}=\sum_{|\mathbf{m}| \text { even }} \llbracket \nu \rrbracket_{\mathbf{m}} K^{\mathbf{m}}(x, e)=\sum_{|\mathbf{m}| \text { even }} d_{\mathbf{m}} \frac{\Perp^{2} \rrbracket_{\mathbf{m}}}{(d / r)_{\mathbf{m}}} \phi^{\mathbf{m}}(x)$,

where the coefficients $\llbracket \nu \rrbracket_{\mathbf{m}}$ have not yet been explicitly computed in the higher rank case. It follows that

$$
c_{\mathbf{m}}^{\nu}=d_{\mathbf{m}} \frac{\llbracket \nu \rrbracket_{\mathbf{m}}}{(d / r)_{\mathbf{m}}}
$$


for all partitions $\mathbf{m}$ with $|\mathbf{m}|$ even. By [11, Section XI.4], the respective norms satisfy

$$
\frac{\left\|\phi^{\mathbf{m}}\right\|_{F}^{2}}{\left\|\phi^{\mathbf{m}}\right\|_{\nu}^{2}}=(\nu)_{\mathbf{m}} .
$$

Combining (115), (119) and (120) implies the assertion, namely

$$
b_{\mathbf{m}}^{\nu}=\frac{d_{\mathbf{m}}}{(\nu)_{\mathbf{m}}} \frac{\left\lfloor\nu \Perp_{\mathbf{m}}\right.}{(d / r)_{\mathbf{m}}} .
$$

In the complex case (116) and (109) yield the Peter-Weyl component

$$
\frac{d_{\mathbf{m}}}{\left(d_{X} / r\right)_{\mathbf{m}}} \frac{1}{(\nu)_{m}} \phi_{\mathbf{C}}^{\mathbf{m}}(\nabla)=\frac{1}{(\nu)_{\mathbf{m}}} K^{\mathbf{m}}(\partial, \partial)
$$

and we indeed recover the Arazy-Ørsted formula (105).

Note that in course of the proof we have obtained also the following "real analogue" of the Faraut-Korányi formula - namely, the Peter-Weyl decomposition (under $K^{\mathbf{C}}$ ) of the holomorphic function $I(z)=h\left(z, z^{\#}\right)^{-\nu / 2}$.

Theorem 15. We have

$$
I(z)=\sum_{\mathbf{m}} \llbracket \nu \rrbracket_{\mathbf{m}} \frac{d_{\mathbf{m}}}{\left(d_{X} / r\right)_{\mathbf{m}}} \phi_{\mathbf{C}}^{\mathbf{m}}(z),
$$

where $\llbracket \nu \rrbracket_{\mathbf{m}}$ is given by

$$
\llbracket \nu \rrbracket_{\mathbf{m}}=\left(\nu_{\mathbf{R}}\right)_{\mathbf{m}}
$$

for domains not of type (A), including the complex case. Here $\mathbf{m}$ ranges over all signatures of length $r$.

Remark 16. The relationship between the two "Pochhammer symbols"

$$
\{\nu\}_{\mathbf{m}_{\mathbf{C}}}=\frac{1}{b_{\mathbf{m}}^{\nu}}, \quad \llbracket \nu \rrbracket_{\mathbf{m}_{\mathbf{C}}}=\frac{\left(d_{X} / r\right)_{\mathbf{m}}}{d_{\mathbf{m}}} c_{\mathbf{m}}^{\nu},
$$

can be simply stated as

$$
\frac{1}{\{\nu\}_{\mathbf{m}_{\mathbf{C}}}}=\frac{d_{\mathbf{m}}}{\left(d_{X} / r\right)_{\mathbf{m}}} \frac{\llbracket \nu \Perp_{\mathbf{m}_{\mathbf{C}}}}{(\nu)_{\mathbf{m}_{\mathbf{C}}}}
$$

where $(\nu)_{\mathbf{m}_{\mathbf{C}}}=\Gamma_{\mathbf{C}}\left(\nu+\mathbf{m}_{\mathbf{C}}\right) / \Gamma_{\mathbf{C}}(\nu)$ is the ordinary multi-Pochhammer symbol corresponding to the domain $D^{\mathrm{C}}$.

Example 17. Consider $D=(-1,+1) \subset \mathbf{R}$, the unit interval, for which $D^{\mathbf{C}}=\mathbf{D}$, the unit disc in $\mathbf{C}$. Then

$$
B_{\nu} f(0)=c_{\nu} \int_{-1}^{1} f(x)\left(1-x^{2}\right)^{\nu / 2-1} d x .
$$

The integral vanishes if $f$ is an odd function, while

$$
\int_{-1}^{1} x^{2 k}\left(1-x^{2}\right)^{\nu / 2-1} d x=2 \int_{0}^{1} t^{k}(1-t)^{\nu / 2-1} \frac{d t}{2 \sqrt{t}}=\frac{\Gamma\left(k+\frac{1}{2}\right) \Gamma\left(\frac{\nu}{2}\right)}{\Gamma\left(k+\frac{\nu}{2}+\frac{1}{2}\right)} .
$$

Taking in particular $k=0$, it follows that $1 / c_{\nu}=\Gamma\left(\frac{1}{2}\right) \Gamma\left(\frac{\nu}{2}\right) / \Gamma\left(\frac{\nu}{2}+\frac{1}{2}\right)$, so

$$
B_{\nu}\left(x^{2 k}\right)(0)=\frac{\left(\frac{1}{2}\right)_{k}}{\left(\frac{\nu+1}{2}\right)_{k}} .
$$


Finally, we know from the stationary phase method that if $f(x)=O\left(|x|^{k}\right)$, then $B_{\nu} f(0)=O\left(\nu^{-k}\right)$. Thus it is legitimate to simply sum up the contributions (122) corresponding to the each term in the Taylor expansion $f(x)=\sum_{k=0}^{\infty} \frac{f^{(k)}(0)}{k !} x^{k}$ of $f$ to obtain

$$
B_{\nu} f(0)=\sum_{k=0}^{\infty} \frac{f^{(2 k)}(0)}{(2 k) !} \frac{\left(\frac{1}{2}\right)_{k}}{\left(\frac{\nu+1}{2}\right)_{k}} .
$$

Note that this time the signatures are just nonnegative integers $\mathbf{n}=(n)$, and $K=\{ \pm 1\}$; thus even signatures are those for which $n=2 m$ is an even integer, and $\psi_{\mathbf{C}}^{\mathbf{n}}(z)=z^{2 m}$, with $\left\|\psi_{\mathbf{C}}^{\mathbf{n}}\right\|_{F}^{2}=(2 m)$ !.

Combining the last formula with the doubling formula $\Gamma(x) \Gamma\left(x+\frac{1}{2}\right)=2^{1-2 x} \Gamma\left(\frac{1}{2}\right)$. $\Gamma(2 x)$ for the Gamma function, it can also be rewritten as

$$
B_{\nu} f(0)=\sum_{m=0}^{\infty} \frac{f^{(2 m)}(0)}{m ! 4^{m}\left(\frac{\nu+1}{2}\right)_{m}},
$$

in complete agreement with the last theorem. (Note that now $r=r_{\mathbf{C}}=1, d_{Y}=$ $d_{V}=0$, and we can view $D$ as both type $D_{1}$ and $A_{1}$.)

Remark 18. Symbolically, (123) can be written as $\left.B_{\nu}\right|_{0}={ }_{0} F_{1}\left(\frac{\nu+1}{2} \mid \frac{1}{4} \nabla^{2}\right)$. Exploiting $G$-invariance, this gives an expression for $B_{\nu}$ in terms of the invariant Laplacian $\left(\left(1-x^{2}\right) \nabla\right)^{2}$ of $D$.

\section{Concluding Remarks}

6.1 Schwartz-space boundedness. It was shown in Theorem 2 in [8] that (phrased in our current terminology) in the "product" case, any $G$-invariant linear differential operator from functions on $D^{\mathbf{C}}$ into functions on $D$ is automatically continuous from the Schwartz space $\mathcal{S}\left(D^{\mathbf{C}}\right.$ ) (as defined e.g. in [13]) into $\mathcal{S}(D)$. The same argument also works in the general case, thus showing that the assertion is in fact valid even for any real bounded symmetric domain $D$.

6.2 Convergence in various senses. In our Definition 2 and elsewhere, we have always been interested in the pointwise convergence of the various asymptotic expansions such as (87) or (89). On the other hand, for the Toeplitz calculus in the "product" case, (8) was established in [6] not only pointwise (in the sense of integral kernels, i.e. $\left.\left\langle\mathcal{T}_{f} \mathcal{T}_{g} K_{x}, K_{y}\right\rangle \approx \sum_{m=0}^{\infty} \nu^{-m}\left\langle\mathcal{T}_{C_{m}(f, g)} K_{x}, K_{y}\right\rangle\right)$, but in operator norm. (The former, of course, follows from the latter upon a simple application of the Schwarz inequality.)

Is there some analogous stronger sense in which (89) would be true?

The obvious candidate - namely, that (89) hold in the sense of the norms in $\mathcal{H}^{\nu}$ - does not correspond to operator norms in (8) in the "product" case, but rather to the Hilbert-Schmidt norms. It is unclear whether (8) holds true with operator norms replaced by Hilbert-Schmidt norms, even for symbols $f, g$ with compact support (more likely it does not). Hence this is probably a blind alley.

The next thing that comes to mind is to replace the norm convergence by the weak convergence, but then the problem is that the spaces in question vary with $\nu$, so it is unclear with what elements one should form the inner products. (The only natural ones around seem to be the reproducing kernels $K_{x}^{(\nu)}$, but that brings us back to the pointwise convergence again.) 
6.3 General covariant calculi. Throughout the paper, we have been discussing the star-restriction etc. associated to the Toeplitz calculus. Of course the next thing to be done is to extend everything also to other covariant calculi. So far, the only known results - and very partial ones at that - are available for the Weyl calculus; see Unterberger and Unterberger [23] for the "product case" with $D=\mathbf{D}$, the unit disc, and Arazy and Upmeier [4] for the "flat" cases $D=\mathbf{C}^{d}$ and $D=\mathbf{R}^{d}$.

One way of getting such results might be by using the fact that any two covariant calculi on $D$ differ by a $G$-invariant operator. In fact, for any covariant calculus $\mathcal{A}: C^{\infty}(D) \rightarrow \mathcal{H}^{\nu}$ we have $\mathcal{A}_{f}=\mathcal{T}_{M_{\mathcal{A}} f}$, where $M_{\mathcal{A}}:=\left(\mathcal{T}^{*} \mathcal{T}\right)^{-1}\left(\mathcal{T}^{*} \mathcal{A}\right)$ is $G$ invariant, by $(15)$ and $(60)$. (The inverse $\left(\mathcal{T}^{*} \mathcal{T}\right)^{-1}=\left(B_{\nu}\right)^{-1}$ exists since (44) never vanishes; we are of course a bit sloppy here about the boundedness and domains of definition of the various operators.) Hence, if $\# \mathcal{A}, \mathcal{B}$ is the star-restriction operator with respect to a covariant calculus $\mathcal{A}$ on $D^{\mathbf{C}}$ and another covariant calculus $\mathcal{B}$ on $D$, that is,

$$
\mathcal{B}_{\#_{\mathcal{A}, \mathcal{B}} F}=\mathcal{A}_{F} I
$$

then

$$
\mathcal{T}_{M_{\mathcal{B}} \#_{\mathcal{A}, \mathcal{B}} F}=\mathcal{B}_{\# \mathcal{A}, \mathcal{B} F}=\mathcal{A}_{F} I=\mathcal{T}_{M_{\mathcal{A}}^{\mathrm{C}} F}^{\mathrm{C}} I=\mathcal{T}_{\#\left(M_{\mathcal{A}}^{\mathrm{C}} F\right)}
$$

(where the last \# is the one with respect to $\mathcal{T}^{\mathbf{C}}$ and $\mathcal{T}$, which we have been working with throughout this paper); that is,

$$
\#_{\mathcal{A}, \mathcal{B}}=\left(M_{\mathcal{B}}\right)^{-1} \# M_{\mathcal{A}}^{\mathbf{C}}
$$

(provided $M_{\mathcal{B}}$ is invertible). In this sense, all possible star-restrictions are conjugate to the Toeplitz one via $G$ - and $G^{\mathbf{C}}$-invariant operators (on the left and on the right, respectively). This approach was, for instance, used in [4], Proposition 2.1 and 5.1, for reducing the Weyl calculus (and other "one-parameter calculi" studied there) to the Toeplitz one in the "flat" cases $D=\mathbf{C}^{d}, \mathbf{R}^{d}$, and in [8]. It is, however, unclear (in addition to the matter of the invertibility of $M_{\mathcal{B}}$ ) whether this approach will really be useful in the general case, since for instance the "factorization property" from Proposition 5 will in general be destroyed by the conjugation with $M_{\mathcal{B}}$ and $M_{\mathcal{A}}^{\mathrm{C}}$, and thus for our results from Section 4 completely different methods seem to be needed.

6.4 The flat case. The results of Sections 3-5 easily extend also to the "flat" case $D=\mathbf{R}^{d}$. In fact, the integrals (83) become simply

$$
\begin{aligned}
B_{\nu}^{\mathbf{C}} F(z) & =\left(\frac{\nu}{\pi}\right)^{d} \int_{\mathbf{C}^{d}} F(w) e^{-\nu|z-w|^{2}} d w \\
B_{\nu} f(x) & =\left(\frac{\nu}{\pi}\right)^{d / 2} \int_{\mathbf{R}^{d}} f(\zeta) e^{-\nu(x-\zeta)^{2} / 2} d \zeta,
\end{aligned}
$$

i.e. just the heat solution operators (66), (48), while (92) becomes

$$
\mathcal{T}^{*} \mathcal{T}_{F}^{\mathbf{C}} I(0)=\left(\frac{\nu}{\pi}\right)^{d} \int_{\mathbf{C}^{d}} F(w) e^{\nu w^{2} / 2-\nu|w|^{2}} d w,
$$

where $w^{2}:=\sum_{j=1}^{d} w_{j}^{2}$. By easy manipulations, this can again be reduced to heat solution operators. In Section 5 , signatures $\mathbf{m}=(m)$ reduce just to a single integer $m \geq 0$, and the Peter-Weyl space $\mathcal{P}^{\mathbf{m}}$ consists of all homogeneous polynomials of degree $m$. The even signatures are the even numbers $m$, and $\phi_{\mathbf{C}}^{(2 m)}(x)=\left(\sum_{j=1}^{d} x_{j}^{2}\right)^{m}$. 
Finally, since

$$
B_{\nu}=e^{\Delta / 2 \nu}=\sum_{m=0}^{\infty} \frac{\Delta^{m}}{m ! 2^{m} \nu^{m}}=\sum_{m=0}^{\infty} \frac{\phi^{(2 m)} \mathbf{c}(\nabla)}{m ! 2^{m} \nu^{m}}
$$

we get

$$
\left(\left(B_{\nu}\right)_{(2 m)}\right)_{0}=\frac{\phi_{\mathbf{C}}^{(2 m)}(\nabla)}{\{\nu\}_{(2 m)}}
$$

with

$$
\{\nu\}_{(2 m)}=m ! 2^{m} \nu^{m} .
$$

Of course, this formula can likewise be derived along the lines of the proof of Theorem 14.

We leave the complex case $D=\mathbf{C}^{d}$ to the reader.

6.5 Another Weyl calculus. For the "flat product case" $D=\mathbf{C}^{d}$, it is known that the Toeplitz calculus $f \mapsto \mathcal{T}_{f}$ maps $L^{2}\left(d \mu_{0}\right)$ boundedly into the space of Hilbert-Schmidt operators. One can therefore consider its polar decomposition $\mathcal{T}=\mathcal{V}\left(\mathcal{T}^{*} \mathcal{T}\right)^{1 / 2}=\mathcal{V}\left(B_{\nu}\right)^{1 / 2}$, where $\mathcal{V}$ is a partial isometry with initial space $\operatorname{Ran} \mathcal{T}^{*}=(\operatorname{Ker} \mathcal{T})^{\perp}$ and final space $\operatorname{Ran} \mathcal{T}=\left(\operatorname{Ker} \mathcal{T}^{*}\right)^{\perp}$; that is, as both $\mathcal{T}$ and $\mathcal{T}^{*}$ are injective, $\mathcal{V}$ is a unitary operator from $L^{2}\left(\mathbf{C}^{d}\right)$ onto the Hilbert-Schmidt operators on $\mathcal{H}^{\nu}\left(\mathbf{C}^{d}\right)$. It turns out that $\mathcal{V}$ coincides with, surprisingly, our old friend $\mathcal{W}$, the Weyl calculus on $D=\mathbf{C}^{d}$. This leads naturally to the interesting question of determining the partial-isometric part of the polar decomposition of $\mathcal{T}$ also in the general case of any real bounded symmetric domain $D$. Since $\mathcal{T}$ and $\mathcal{T}^{*}$ can easily be shown to be still injective, this partial-isometric part $\mathcal{V}$ will again in fact be a unitary operator from $L^{2}\left(d \mu_{0}\right)$ onto $\mathcal{H}^{\nu}\left(D^{\mathbf{C}}\right)$.

For $D=\mathbf{R}^{d}$, the real "flat" case, it was shown in [21] that $\mathcal{V}$ is actually the Bargmann transform from $L^{2}\left(\mathbf{R}^{d}\right)$ onto $\mathcal{H}^{\nu}\left(\mathbf{C}^{d}\right)$. For the "product case" $D^{\mathbf{C}}=$ $D \times \bar{D}$, with $D$ a complex bounded symmetric domain, the operator $\mathcal{V}$ was studied by Ørsted and Zhang [22], and later christened to the "generalized Segal-Bargmann transform"; in any case it turned out to be different from the Weyl calculus on $D$ (except for $D=\mathbf{C}^{d}$ ).

For real bounded symmetric domains other than the "flat" or "product" cases, the operator $\mathcal{V}$ seems not to have been hitherto investigated.

\section{REFERENCES}

[1] J. Arazy, B. Ørsted: Asymptotic expansions of Berezin transforms, Indiana Univ. Math. J. 49 (2000), 7-30.

[2] J. Arazy, H. Upmeier: Invariant symbolic calculi and eigenvalues of invariant operators on symmetric domains, Function spaces, interpolation theory, and related topics (Lund, 2000) (A. Kufner, M. Cwikel, M. Engliš, L.-E. Persson, and G. Sparr, eds.), pp. 151-211, Walter de Gruyter, Berlin, 2002.

[3] J. Arazy, H. Upmeier: Covariant symbolic calculi on real symmetric domains, Singular integral operators, factorization and applications, pp. 1-27, Oper. Theory Adv. Appl. 142, Birkhauser, Basel, 2003.

[4] J. Arazy, H. Upmeier: A one-parameter calculus for symmetric domains, Math. Nachr. 280 (2007), 939-961.

[5] F.A. Berezin: Quantization, Math. USSR Izvestiya 8 (1974), 1109-1163.

[6] D. Borthwick, A. Lesniewski, H. Upmeier: Non-perturbative deformation quantization on Cartan domains, J. Funct. Anal. 113 (1993), 153-176.

[7] G. v. Dijk, M. Pevzner: Berezin kernels of tube domains, J. Funct. Anal. 181 (2001), 189-208. 
[8] M. Engliš: Berezin-Toeplitz quantization and invariant symbolic calculi, Lett. Math. Phys. 65 (2003), 59-74.

[9] M. Engliš: Berezin-Toeplitz quantization on the Schwartz space of bounded symmetric domains, J. Lie Theory 15 (2005), 27-50.

[10] M. Engliš: Toeplitz operators and group representations, J. Fourier Anal. Appl. 13 (2007), $243-265$.

[11] J. Faraut, A. Korányi: Analysis on symmetric cones, Clarendon Press, Oxford, 1994.

[12] G.B. Folland: Harmonic analysis in phase space, Ann. Math. Studies vol. 122, Princeton University Press, Princeton, 1989.

[13] R. Gangolli, V.S. Varadarajan, Harmonic analysis of spherical functions on real reductive groups, Springer, New York, 1985.

[14] S. Helgason: Differential geometry and symmetric spaces, Academic Press, New YorkLondon, 1962.

[15] L. Hörmander: The analysis of linear partial differential operators, vol. I, Grundlehren der mathematischen Wissenschaften, vol. 256, Springer-Verlag, Berlin-Heidelberg-New York, 1985.

[16] L.K. Hua: Harmonic analysis of functions of several complex variables in the classical domains, Amer. Math. Soc., Providence, 1963.

[17] W. Kaup: On real Cartan factors, Manuscr. Math. 92 (1997), 191-222.

[18] O. Loos: Bounded symmetric domains and Jordan pairs, University of California, Irvine, 1977.

[19] I.G. MacDonald: Symmetric functions and Hall polynomials (2nd edition), Clarendon Press, Oxford, 1995.

[20] Y. Neretin: Plancherel formula for Berezin deformation on $L^{2}$ on Riemann symmetric space, J. Funct. Anal. 189 (2002), 336-408.

[21] G. Olafsson, B. Ørsted: Generalizations of the Bargmann transform, Lie theory and its applications in physics (Clausthal 1995), pp. 3-14, World Scientific, River Edge, 1996.

[22] B. Ørsted, G. Zhang: Weyl quantization and tensor products of Fock and Bergman spaces, Indiana Univ. Math. J. 43 (1994), 551-582.

[23] A. Unterberger, J. Unterberger: La série discrète de $S L(2, \mathbf{R})$ et les opérateurs pseudodifférentiels sur une demi-droite, Ann. Sci. École Norm. Sup. (4) 17 (1984), 83-16.

[24] A. Unterberger, H. Upmeier: Berezin transform and invariant differential operators, Comm. Math. Phys. 164 (1994), 563-598.

[25] H. Upmeier: Toeplitz operators on bounded symmetric domains, Trans. Amer. Math. Soc. 280 (1983), 221-237.

[26] G. Zhang: Berezin transform on real bounded symmetric domains, Trans. Amer. Math. Soc. 353 (2001), 3769-3787.

[27] G. Zhang: Branching coefficients of holomorphic representations and Segal-Bargmann transform, J. Funct. Anal. 195 (2002), 306-349.

[28] G. Zhang: Degenerate principal series representations and their holomorphic extensions, preprint (2007), arXiv:math/0711.1480.

Mathematics Institute, Silesian University at Opava, Na RybníčKu 1, 74601 Opava, Czech Republic and Mathematics Institute, Žitná 25, 11567 Prague 1, Czech Republic

E-mail address: englis@math.cas.cz

Fachbereich Mathematik, Universität Marburg, D-35032 Marburg, Germany

E-mail address: upmeier@mathematik.uni-marburg.de 